\title{
Symmetric diblock copolymer thin films confined between homogeneous and patterned surfaces: Simulations and theory
}

\author{
Qiang Wang, Shyamal K. Nath, ${ }^{\text {a) }}$ Michael D. Graham, Paul F. Nealey, \\ and Juan J. de Pablob) \\ Department of Chemical Engineering, University of Wisconsin-Madison, Madison, Wisconsin 53706-1691
}

(Received 15 November 1999; accepted 13 March 2000)

\begin{abstract}
We have investigated the ability of a simple phenomenological theory to describe the behavior of symmetric diblock copolymer thin films confined between two hard surfaces. Prior knowledge of the morphology in the confined films is crucial for applying this theory to predict the phase diagram of such systems. Taking advantage of our observations in Monte Carlo simulations, we use the theory to construct phase diagrams for thin films confined between patterned-homogeneous surfaces, and obtain good agreement with our results of simulations. Two conditions are essential for obtaining long-range ordered perpendicular lamellae: a lower stripe-patterned surface with the surface pattern period $L_{s}$ comparable to the bulk lamellar period $L_{0}$, and an upper neutral or weakly preferential surface. We have also examined the undulation of perpendicular lamellae between two hard surfaces. For the cases of two homogeneous (preferential) surfaces and patterned-preferential surfaces, our calculations using the phenomenological theory indicate that the amplitudes of the undulation are on the same order of magnitude as observed in our Monte Carlo simulations, and are one order of magnitude larger than previously reported. The theory, however, is unable to capture the shape of the undulation. For the case of patterned-neutral surfaces, we find that an earlier analysis is unable to yield the undulations that would stabilize the perpendicular lamellar morphology. We have addressed this issue and obtained undulations that are consistent with our observations from Monte Carlo simulations. (C) 2000 American Institute of Physics.
\end{abstract}

[S0021-9606(00)70322-9]

\section{INTRODUCTION}

Recently, the study of diblock copolymer thin films has attracted significant interest because of their potential applications in nanofabrication. Much of the research in this area has been carried out on symmetric diblock copolymers, which in the bulk form lamellar structures at temperatures below the order-disorder transition (ODT). For some applications (e.g., nanolithography), it is desirable to generate macroscopically ordered (over microns) lamellar structure perpendicular to a substrate. The purpose of this work is to investigate under which circumstances such structures can occur.

It has been established that for symmetric diblock copolymer thin films confined between two strongly preferential surfaces, the equilibrium morphology consists of lamellae oriented parallel to the surfaces, with the preferred blocks segregating to the corresponding surfaces. ${ }^{1-11}$ This reduces the surface-block interfacial energy. In the case of parallel lamellae confined between two hard (impenetrable) surfaces, the confined lamellar period $L$ may vary from the bulk value $L_{0}$ to accommodate the frustration between surface separation $D$ and $L_{0}$. Such stretching or compressing of lamellae is accompanied by an elastic free energy penalty associated with chain conformational entropy.

As surface preference becomes less specific, an addi-

\footnotetext{
${ }^{a)}$ Current address: Molecular Simulations Inc., 9685 Scranton Road, San Diego, California 92121.

b) Author to whom correspondence should be addressed.
}

tional increase in the frustration between $D$ and $L_{0}$ can lead to a perpendicular orientation of the lamellae with $L \approx L_{0} \cdot{ }^{4,5,8-18}$ Since in this case both $\mathrm{A}$ and $\mathrm{B}$ blocks are present near the surfaces, the surface-block interfacial energy can be relatively high if the surfaces are not completely neutral, which is often the case in experiments. It has been pointed out ${ }^{5,9-11,17-19}$ that in perpendicular lamellae confined between two homogeneous and (weakly) preferential surfaces, undulations of the A-B interface can be caused by the surface-induced segregation of preferred blocks near the surfaces; these could reduce the surface-block interfacial energy and thus stabilize the perpendicular morphology.

Perpendicular lamellae have been observed experimentally between two homogeneous surfaces when the surface preference is neutral or weak; transmission electron microscopy (TEM) or field emission scanning electron microscopy (FESEM) images show that the orientation of such lamellae is short-ranged (tens to hundreds of nanometers). ${ }^{4,12,13}$ More recently, experiments, ${ }^{20}$ Monte Carlo simulations, ${ }^{21}$ and theoretical calculations ${ }^{22-30}$ have shown that macroscopically ordered perpendicular lamellae can be obtained by depositing symmetric diblock copolymers on stripe-patterned chemically heterogeneous surfaces, where the surface pattern period $L_{s}$ is comparable to the bulk lamellar period $L_{0}$.

To better understand the behavior of symmetric diblock copolymer thin films confined between two hard surfaces, we have performed extensive Monte Carlo simulations that have provided us with valuable insights regarding the morphology of such systems. ${ }^{5,21}$ The simulation results have served as a 
guide to propose plausible solutions for theoretical calculations. In this work we examine the ability of a simple phenomenological theory to describe the behavior of symmetric diblock copolymer thin films confined between two hard surfaces. For a given morphology of the system in the strong segregation limit, this theory can be used to estimate the free energy in terms of elastic contributions (associated with chain conformational entropy), block-block interfacial contributions, surface-block interfacial contributions, and bending contributions (if the A-B interfaces in lamellae are not flat). Our hope is that such a theory could be used to rationalize and describe the results of our recent simulations of confined copolymers.

Various forms of the phenomenological theory considered in this work for symmetric diblock copolymers have appeared in the literature. ${ }^{31-35}$ Turner built on earlier work on this theory by adding surface-block interaction terms to study the phase diagram of symmetric diblock copolymer thin films confined between two homogeneous surfaces, ${ }^{7}$ where he only considered the parallel symmetric and antisymmetric lamellar morphology between the two surfaces. ${ }^{7}$ Walton et al. extended Turner's study by considering the perpendicular lamellar morphology. ${ }^{8}$ Independently, Kikuchi and Binder proposed a similar theory to compare the stability of parallel symmetric lamellae and perpendicular lamellae between two identical (homogeneous) surfaces. ${ }^{15}$ For similar systems, Matsen ${ }^{11}$ and Geisinger et al. ${ }^{17}$ compared the predictions of this theory with self-consistent field calculations and found only qualitative agreement. The phenomenological theory does not take hard-surface effects ${ }^{5}$ into account, and is unable to predict in a unique manner the formation of perpendicular lamellae between neutral surfaces. ${ }^{36}$

Prior knowledge of the morphology in confined films is crucial for applying the phenomenological theory. Starting from a random (disordered) state, our Monte Carlo simulations for patterned-homogeneous surfaces ${ }^{21}$ have yielded various types of morphology (depending on the surface configuration), thereby providing the essential information for applying the theory. Recently, Pereira and Williams used that theory to calculate phase diagrams for symmetric diblock copolymer thin films confined between patternedhomogeneous surfaces. ${ }^{27-29}$ Some types of morphology observed in our recent Monte Carlo simulations, however, were not considered in their work. Based on the observations from such simulations, ${ }^{21}$ in this work we use the phenomenological theory to construct the phase diagram of symmetric diblock copolymer thin films confined between patternedhomogeneous surfaces.

In related work, Pereira and Williams used the same theory to examine the undulations of A-B interfaces in perpendicular lamellae confined between two homogeneous and preferential surfaces, ${ }^{19}$ patterned-preferential surfaces, ${ }^{37}$ and patterned-neutral surfaces. ${ }^{38}$ Such undulations are caused by the preference of the homogeneous surface to one of the two blocks, or the incommensuration between $L_{s}$ and $L_{0}$, both of which are difficult to control in experiments. The undulation could have adverse effects for applications in nanolithography. The phenomenological theory considered here involves a number of simplifications and approximations which are necessary to render its solutions tractable. It is therefore of interest to revisit Pereira and Williams' calculations, and compare the results of theoretical predictions with those of Monte Carlo simulations ${ }^{5,21}$ which are more computationally demanding but are free of the simplifications and approximations of the theory. We find that for some systems our findings differ from those reported in earlier work.

\section{MODELS}

\section{A. Phenomenological theory}

In the strong segregation limit, where lamellar structures of period $L$ are well-developed and the thickness of A-B interfaces is vanishingly small, the free energy of symmetric diblock copolymer thin films is assumed to consist of four contributions: the elastic free energy of the lamellae (associated with chain conformational entropy), the block-block interfacial energy, the surface-block interfacial energy, and the bending free energy of the lamellae if the A-B interfaces in the lamellae are not flat. The elastic free energy per diblock copolymer chain in the lamellae of period $L$ can be written as

$$
f_{e l}=a L^{2},
$$

where $a$ is a constant whose value depends on the total number of segments in a diblock copolymer chain (denoted by $N$ here) and the distribution of the chain ends in the lamellae. According to Semenov, who assumed a distribution of chain ends that minimizes the elastic free energy of the inner region of a micelle, ${ }^{31} a$ is found to be

$$
a=\frac{\pi^{2} k_{B} T}{32 N l^{2}},
$$

where $k_{B}$ is the Boltzmann constant, $T$ the absolute temperature, and $l$ the Kuhn length of a segment. By assuming that chains terminate at the interfaces between adjacent chain layers, Semenov ${ }^{31}$ and others ${ }^{7,32}$ have found

$$
a=\frac{3 k_{B} T}{8 N l^{2}} .
$$

As pointed out by Semenov, using Eq. (3) instead of Eq. (2) raises the elastic free energy by a factor of $12 / \pi^{2} .{ }^{31}$ Note, however, that as we shall see below the value of $a$ does not affect the dimensionless free energy of the system and the dimensionless differential equations derived later. For a diblock copolymer with different end-to-end distances of the A and B blocks, denoted by $D_{\mathrm{A}}$ and $D_{\mathrm{B}}$ respectively, Eq. (1) becomes

$$
f_{e l}=8 a\left(D_{\mathrm{A}}^{2}+D_{\mathrm{B}}^{2}\right) .
$$

The A-B interfacial energy per chain in lamellae of period $L$ can be calculated from the block-block interfacial tension $\sigma_{\mathrm{AB}}>0$ according to

$$
f_{A B}=\frac{2 \sigma_{\mathrm{AB}}}{\rho L},
$$




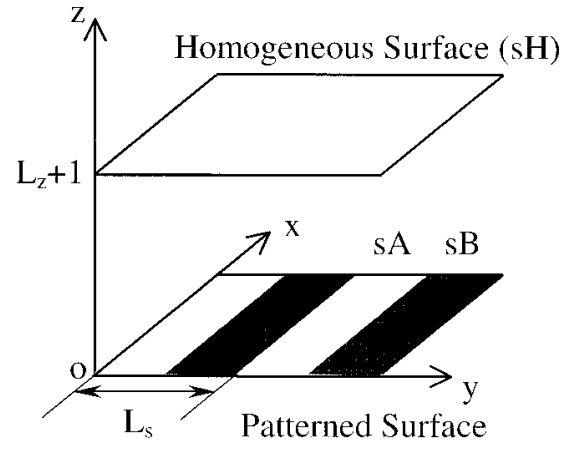

FIG. 1. Configuration of the patterned-homogeneous surfaces. Both surfaces are hard and flat. The stripes on the lower patterned surface have the same width $L_{s} / 2$ and alternatively prefer A and B blocks of the copolymer (denoted by $\mathrm{sA}$ and $\mathrm{sB}$ stripes, respectively). The upper homogeneous surface is either neutral or preferential to one of the two blocks.

where $\rho$ is the number density of chains in the system, and where it is assumed that A-B interfaces are flat. Surfaceblock interfacial energies can be calculated from the corresponding surface-block interfacial tensions in a similar way.

In the strong segregation limit, the characteristic period of lamellae in the bulk, $L_{0}$, can be obtained by minimizing with respect to $L$ the total free energy per chain in the bulk, which consists of the elastic free energy given by Eq. (1) and the A-B interfacial energy given by Eq. (5). This leads to

$$
L_{0}=\left(\frac{\sigma_{\mathrm{AB}}}{a \rho}\right)^{1 / 3} .
$$

Note that both Eqs. (2) and (3) give $a \propto 1 / N$. As in Ref. 32, by assuming a constant number density of segments $\rho_{s}$ $=\rho N$, we can recover the scaling of the bulk lamellar period $L_{0}$ with chain length $N$, namely, $L_{0} \propto N^{2 / 3}$, which is consistent with experimental findings. ${ }^{39}$ (For large $N, \sigma_{\mathrm{AB}}$ is independent of $N .^{32}$ ) In addition, by substituting Eq. (6) into Eqs. (1) and (5), we get the ratio of the elastic free energy to the A-B interfacial energy in the bulk,

$$
\frac{f_{e l}\left(L_{0}\right)}{f_{A B}\left(L_{0}\right)}=\frac{1}{2} .
$$

Note that this ratio is independent of $a$.

According to Wang, ${ }^{35}$ if the A-B interfaces in a lamellar morphology are not flat, the bending free energy per chain arising from a curvature $c$ can be estimated as

$$
f_{\text {bend }}=\tilde{a} c^{2} L^{4},
$$

where $\tilde{a}=\frac{3}{64} a$.

\section{B. Monte Carlo simulations}

Our Monte Carlo simulations are performed in an expanded grand-canonical ensemble in the framework of a simple cubic lattice. Detailed descriptions can be found in Refs. 5 and 21. Only a brief summary is given here.

A rectangular simulation box of dimensions $L_{x}, L_{y}$, and $L_{z}$ is employed. Periodic boundary conditions are imposed in the $x$ and $y$ directions. Two flat surfaces are introduced through the lattice sites at $z=0$ and $z=L_{z}+1$, respectively (see Fig. 1). These lattice sites are not allowed to be occupied by polymer segments. Diblock copolymers are therefore confined to a thin-film geometry of thickness $D=L_{z}-1$.

In the simulations, we only consider repulsion between nearest-neighbor $\mathrm{A}-\mathrm{B}$ pairs separated by one lattice unit $\left(\epsilon_{\mathrm{A}-\mathrm{B}}>0\right)$, and we set $\epsilon_{\mathrm{A}-\mathrm{A}}=\epsilon_{\mathrm{B}-\mathrm{B}}=0$. Interactions between vacancies (unoccupied lattice sites) and polymer segments are also set to zero. Three kinds of sites populate the surfaces: $\mathrm{sA}, \mathrm{sB}$, and $\mathrm{sH}$, whose nature depends on the type of surface-block interaction. For simplicity, we set $\epsilon_{\mathrm{sA}-\mathrm{A}}$ $=\epsilon_{\mathrm{sB}-\mathrm{B}}=\epsilon_{\mathrm{sH}-\mathrm{A}}=0, \epsilon_{\mathrm{sA}-\mathrm{B}}>0, \epsilon_{\mathrm{sB}-\mathrm{A}}>0$, and $\epsilon_{\mathrm{sH}-\mathrm{B}} \geqslant 0$. For the case of two homogeneous and preferential surfaces studied in this work, the surfaces consist of either $\mathrm{sA}$ or $\mathrm{sB}$ sites. For the case of patterned-homogeneous surfaces, the upper homogeneous surface consists of sH sites. When $\epsilon_{\mathrm{sH}-\mathrm{B}}=0$, the upper surface is neutral, with no preference for either of the two blocks; when $\epsilon_{\mathrm{sH}-\mathrm{B}}>0$, the upper surface repels B blocks, and is therefore preferential to A blocks. The chemically heterogeneous surfaces are patterned with stripes parallel to the $x$ axis, as shown in Fig. 1. The stripes consist of alternating $\mathrm{sA}$ (light regions) and sB (dark regions) sites. All stripes have the same width $L_{s} / 2$, where $L_{s}$ is the period of the surface pattern.

We perform Monte Carlo simulations in a variant of the expanded grand-canonical ensemble method proposed by Escobedo and de Pablo. ${ }^{40}$ The chemical potential and temperature of the simulated system are specified prior to a simulation. The confined copolymers are therefore in equilibrium with a bulk phase having the same chemical potential and temperature, and the density of the system is allowed to fluctuate during the simulation. In addition to molecule displacements by reptation moves and local (crankshaft and kinkjump) moves, we employ growing/shrinking moves performed four segments at a time to gradually insert/remove chains from the system. To facilitate transitions, configurational bias is used for these growing/shrinking moves, leading to an acceptance rate of about $20 \% .^{5}$ A standard Metropolis algorithm is employed in our simulations. One Monte Carlo step (MCS) consists of $0.8 \times L_{x} \times L_{y} \times L_{z}$ trials of reptation, local and growing/shrinking moves, each of which occurs with the same probability. In general, we discard the first 100000 MCS for equilibration, then make a run of at least 500000 MCS while collecting data every 5 MCS.

We study symmetric diblock copolymers of chain length $N=24$. We set the reduced temperature to be $T^{*}$ $\equiv k_{B} T / \epsilon_{\mathrm{A}-\mathrm{B}}=2.3$. We also set the reduced chemical potential at $\mu^{*} \equiv \mu /\left(k_{B} T\right)=41.5$, where $\mu$ is the chemical potential of the system. These conditions lead to a density of the confined films (percentage of occupied lattice sites) of around 0.8 . In the bulk, the ODT of the diblock copolymer is between $T^{*}=2.8-3.0 ;^{5}$ our system is therefore in the intermediate segregation regime. The characteristic period of lamellae in the bulk under the above conditions was estimated to be $L_{0}=12 .^{5}$ 


\section{RESULTS AND DISCUSSION}

\section{A. Phase diagrams of symmetric diblock copolymer thin films confined between patterned-homogeneous surfaces}

Our Monte Carlo simulations have revealed the existence of various types of morphology in thin films confined between patterned-homogeneous surfaces. ${ }^{21}$ Armed with these results, we construct the phase diagrams of the system using the phenomenological theory described above, and compare them with simulations.

\section{Morphology and free energy of confined films}

Figure 1 shows the configuration of the patternedhomogeneous surfaces. For simplicity, we set the surfaceblock interfacial tensions for the lower stripe-patterned surface to be $\sigma_{\mathrm{sA}-\mathrm{A}}=\sigma_{\mathrm{sB}-\mathrm{B}}=\sigma$, and $\sigma_{\mathrm{sA}-\mathrm{B}}=\sigma_{\mathrm{sB}-\mathrm{A}}=\sigma_{m}>\sigma$. The upper homogeneous surface is either neutral or preferential to one of the two blocks. The interfacial tensions between the homogeneous surface and the two blocks are denoted by $\sigma_{\mathrm{AH}}$ and $\sigma_{\mathrm{BH}}$, respectively. The diblock copolymers are confined between the two surfaces and have a film thickness $D$.

Here we assume that all interfaces in the system are flat, and therefore $f_{\text {bend }}=0$. As shown in Fig. 2, based on our Monte Carlo simulation results, we consider eight types of morphology between the patterned-homogeneous surfaces. These are:

(a) Perpendicular lamellae of bulk period $L_{0}$, denoted by |l|.

(b) Perpendicular lamellae complying with the lower surface pattern, denoted by $\mid \|_{s}$.

(c) Checkerboard morphology, denoted by $+[m]$, where $m$ is the number of layers of chains perpendicular to the surfaces in the confined film. We assume that each chain layer has the same thickness $D / m$.

(d) Mixed morphology of perpendicular lamellae complying with the lower surface pattern near the patterned surface, and perpendicular lamellae of period $L_{0}$ near the upper surface. Such morphology is denoted by \|\|$_{s}-\| \mid$. We use $h$ to denote the ratio of the height of the lower morphology to $L_{0}$. When $L_{s} \neq L_{0}$, the smaller the $h$, the lower the free energy of the $\left|\|_{s}-\right|||$ morphology. However, our simulation results indicate that $h$ in this morphology is almost constant regardless of $D$ and $L_{s} .{ }^{21}$ Therefore, in this paper, we simply specify $h=1 / 4$ for this morphology.

(e) Mixed morphology of one layer of checkerboard near the lower patterned surface, and perpendicular lamellae of period $L_{0}$ near the upper surface. Such morphology is denoted by $+[1]-|| \mid$. We assume that in this morphology the thickness of the checkerboard layer is $L_{0} / 2$.

(f) Parallel lamellae, denoted by $\equiv[m]$. Similar to the $+[m]$ morphology, we assume that each chain layer in the $\equiv[m]$ morphology has the same thickness $D / m$.

(g) Mixed morphology of perpendicular lamellae complying with the lower surface pattern near the patterned surface, and parallel lamellae of $m$ layers near the up-

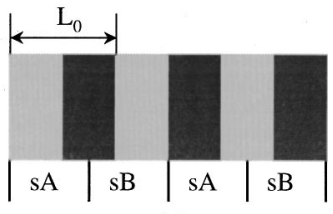

(a)

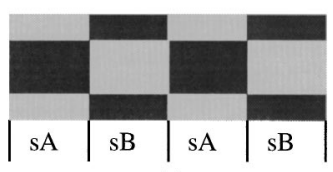

(c)

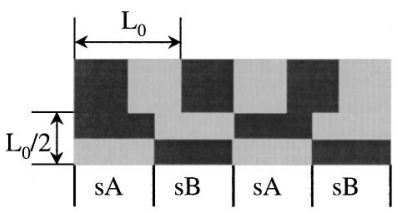

(e)

(g)

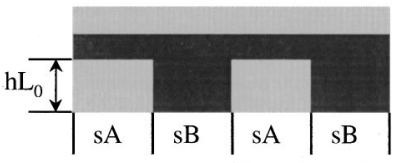

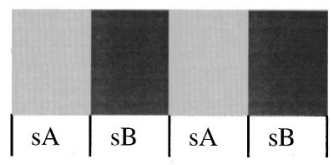

(b)

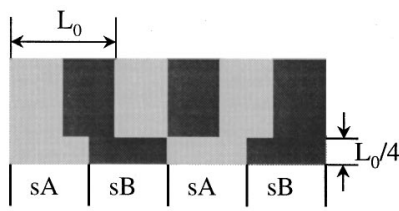

(d)

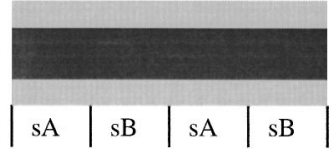

(f)

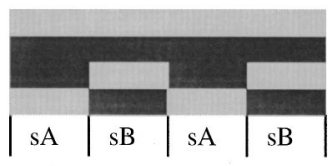

(h)
FIG. 2. Morphology between the patterned-homogeneous surfaces $\left(L_{s} / L_{0}\right.$ $=1.5$ in this figure). Light regions represent A blocks, and dark regions represent B blocks. (a) Perpendicular lamellae of bulk period $L_{0}$, denoted by $I I$; (b) Perpendicular lamellae complying with the surface pattern, denoted by \|\|$_{s}$; (c) Checkerboard morphology, denoted by $+[m]$, where $m$ is the number of the layers of chains perpendicular to the surfaces in the confined film (here $m=2$ ); (d) Mixed morphology of \|\|$_{s}$ near the patterned surface and || $\mid$ near the homogeneous surface, denoted by \|\|$_{s} \mid \|$; (e) Mixed morphology of $+[1]$ near the patterned surface and $\|||$ near the homogeneous surface, denoted by $+[1]$-||l; (f) Parallel lamellae, denoted by $\equiv[m]$ (here $m=2)$; $(\mathrm{g})$ Mixed morphology of \|\|$_{s}$ near the patterned surface and $\equiv[m]$ near the homogeneous surface, denoted by \|\|$_{s^{-}} \equiv[m]$ (here $m=2$ ); (h) Mixed morphology of $+[1]$ near the patterned surface and $\equiv[m-1]$ near the homogeneous surface, denoted by $+[1]-\equiv[m-1]$ (here $m=2$ ).

per surface. Such morphology is denoted by \|\|$_{s^{-}}$ $\equiv[m]$. Again, we denote the height of the lower morphology by $h L_{0}$, and assume that the thickness of each chain layer in the parallel lamellae is $\left(D-h L_{0}\right) / m$. According to our simulation results, ${ }^{21}$ we specify $h$ $\geqslant 1 / 4$ for this morphology.

(h) Mixed morphology of one layer of checkerboard near the lower patterned surface, and parallel lamellae of $m-1$ layers near the upper surface. We assume that the thickness of each chain layer in both the checkerboard and the parallel lamellae is $D / m$. Such morphology is denoted by $+[1]-\equiv[m-1]$. To distinguish it from the $+[m]$ morphology, we specify $m \geqslant 2$ for the $+[1]-\equiv[m-1]$ morphology.

Note that when $L_{s} / L_{0}=1$, the three types of morphology $\|$, \|\|$_{s}$, and $\|\left.\right|_{s^{-}}|| \mid$become identical; we use $\|\left.\right|_{s}$ to represent the morphology.

To calculate the interfacial energy between two mismatched structures with different periods $L_{0}$ and $L_{s}$ (for example, the surface-block interfacial energy between the 
TABLE I. Mismatch ratio $q<1 / 2$ for several values of $L_{s} / L_{0}$.

\begin{tabular}{lc}
\hline \hline$L_{s} / L_{0}$ & $q$ \\
\hline 1 & 0 \\
1.4 & $17 / 35$ \\
1.8 & $22 / 45$ \\
2.2 & $27 / 55$ \\
2.6 & $32 / 65$ \\
3 & $1 / 3$ \\
3.4 & $42 / 85$ \\
3.8 & $47 / 95$ \\
4.2 & $52 / 105$ \\
4.6 & $57 / 115$ \\
5 & $2 / 5$ \\
\hline \hline
\end{tabular}

lower patterned surface and the lamellae when the || $\mid$ morphology forms), we define a mismatch ratio $q$ that represents the average mismatch area per unit length (along the $y$ direction). The value of $q$ depends on $L_{s} / L_{0}$ and the alignment of the two structures. Note that $\left.q\right|_{L_{s} / L_{0}}=\left.q\right|_{L_{0} / L_{s}}$. Theoretical calculations of $q$ are somewhat involved, particularly when $L_{s} / L_{0}$ is an irrational number, or when different alignments of the two structures are considered. In this paper we calculate the value of $q$ for $L_{s} / L_{0}=1 \sim 5$ with a step of 0.0002 , and we only consider the "left" alignment of the two structures of which the first half-periods match with each other [refer to Figs. 2(a), 2(d), and 2(e)]. We find that $q=1 / 2$ for most values of $L_{s} / L_{0}$, except those listed in Table I. Obviously, the larger the $q$, the higher the interfacial energy. In some of our Monte Carlo simulations of thin films confined between patterned-neutral surfaces, ${ }^{21}$ we observed the transposed perpendicular lamellae, where $q=1 / 2$. Therefore, $1 / 2$ can be considered as the upper limit of $q$ for the purposes of our study. To simplify our calculations, we assume that $q$ $=1 / 2$ for all values of $L_{s} / L_{0} \in\left[\frac{1}{5}, 5\right]$, except that $q=0$ at $L_{s} / L_{0}=1, q=1 / 3$ at $L_{s} / L_{0}=1 / 3$ and 3 , and $q=2 / 5$ at $L_{s} / L_{0}=1 / 5$ and 5 . As we shall see below, these "unusual", values of $q$ cause some "spikes" in the phase diagrams.

Table II lists the phenomenological free energies for different types of morphology in the films confined between patterned-homogeneous surfaces with $D / L_{0}>1 / 2$, expressed in the dimensionless form $\left[f_{M}-\left(\sigma_{\mathrm{AH}}+\sigma\right) /(D \rho)\right] / f_{e l}\left(L_{0}\right)$, where $f_{M}$ is the free energy per chain corresponding to the morphology $M$, and $f_{e l}\left(L_{0}\right)=a L_{0}^{2}$ is the elastic free energy per chain in the bulk given by Eq. (1). We define the dimensionless surface pattern period $p \equiv L_{s} / L_{0}$, the dimensionless film thickness $d \equiv D / L_{0}$, and two dimensionless parameters, $\delta_{\mathrm{P}} \equiv\left(\sigma_{m}-\sigma\right) / \sigma_{\mathrm{AB}}>0$ and $\delta_{\mathrm{H}} \equiv\left(\sigma_{\mathrm{BH}}-\sigma_{\mathrm{AH}}\right) / \sigma_{\mathrm{AB}}$. Without loss of generality, we assume that $\delta_{\mathrm{H}} \geqslant 0$, and that A blocks segregate to the upper surface when parallel lamellae form near the upper preferential surface (i.e., in the morphology $\equiv[m],\|\|_{s^{-}} \equiv[m]$, and $\left.+[1]-\equiv[m-1]\right)$. This is consistent with our Monte Carlo simulation results. ${ }^{21}$ Note that the $a$ in Eq. (1) does not appear in these dimensionless free energies. Therefore, its value does not affect the phase diagrams constructed below.

\section{Phase diagram for thin films confined between patterned-neutral surfaces}

For thin films confined between patterned-neutral surfaces, the only types of morphology that were observed in
TABLE II. Phenomenological free energy expressions for different types of morphology in confined films between patterned-homogeneous surfaces with $D / L_{0}>1 / 2$.

\begin{tabular}{cl}
\hline \hline Morphology $(M)$ & \multicolumn{1}{c}{$\left[f_{M}-\frac{\left(\sigma_{A H}+\sigma\right)}{D \rho}\right] / f_{e l}\left(L_{0}\right)$} \\
\hline$\| \mid$ & $\frac{\delta_{\mathrm{H}}}{2 d}+3+\frac{\delta_{\mathrm{P}} q}{d}$ \\
\|\|$_{s}$ & $\frac{\delta_{\mathrm{H}}}{2 d}+\frac{2}{p}+p^{2}$ \\
$+[m]$ & $\frac{\delta_{\mathrm{H}}}{2 d}+\frac{m}{d}+\frac{4 d^{2}}{m^{2}}+\frac{2}{p}$ \\
$+[1]-\|\|$ & $\frac{\delta_{\mathrm{H}}}{2 d}+3+\frac{q}{d}+\frac{p^{2}-3}{4 d}+\frac{1}{2 p d}$ \\
$\equiv[m]$ & $\frac{\delta_{\mathrm{H}}}{2 d}+3+\frac{q}{d}+\frac{1}{p d}$ \\
\|\|$_{s^{-}} \equiv[m]$ & $\frac{m}{d}+\frac{4 d^{2}}{m^{2}}+\frac{\delta_{\mathrm{P}}}{2 d}$ \\
& $\frac{m}{d}+\frac{4 d^{2}}{m^{2}}+\frac{1}{2 d}+\frac{h}{d}\left(p^{2}+\frac{2}{p}\right)-\frac{4 h}{m^{2}}\left[3(d-h)+\frac{h^{2}}{d}\right]$ \\
& $(h \geqslant 1 / 4)$ \\
$+[1]-\equiv[m-1]$ & $\frac{m}{d}+\frac{4 d^{2}}{m^{2}}+\frac{1}{2 d}+\frac{2}{m p}$ \\
& \\
\hline &
\end{tabular}

our Monte Carlo simulations are \|\|$,\|\|_{s^{-}}\|\|,+[m],+[1]-$ $\|$, and \|\|$_{s} \cdot{ }^{21}$ Therefore, we only consider these five types of morphology to construct the phase diagram.

To establish a connection between the phenomenological theory and our Monte Carlo simulations, ${ }^{21}$ we set $\delta_{\mathrm{P}}=2$ and $\delta_{\mathrm{H}}=0$. We vary $p$ within $\left[\frac{1}{5}, 5\right]$ and $d$ within $\left(\frac{1}{2}, 6\right]$ in the phase diagram. For the $+[m]$ morphology, $m$ is chosen to be the positive integer that minimizes the free energy of the system. The calculated phase diagram is shown in Fig. 3. Note that the || $\mid$ morphology coexists at the boundary between the $\left\|\left.\right|_{s^{-}} \mid\right\|$and the $+[1]-\|||$ morphology at $p=2$. Because $q=1 / 3$ at $p=3$, the $\| \mid$ morphology also coexists with the $+[1]-|| \mid$ morphology at $p=3$. Furthermore, these two

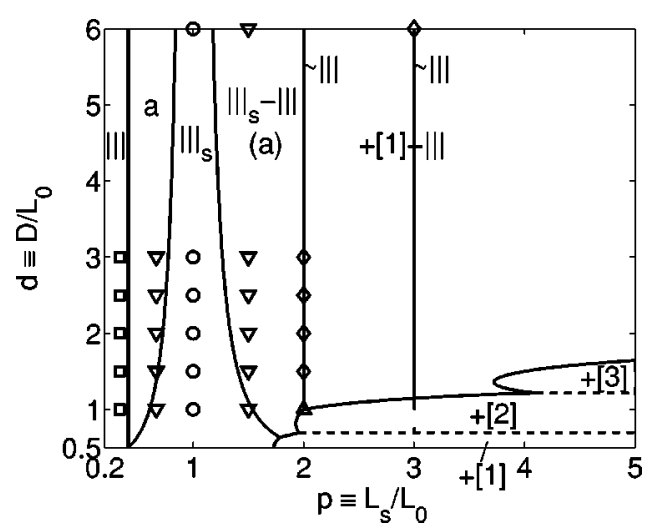

FIG. 3. Phase diagram of symmetric diblock copolymer thin films confined between patterned-neutral surfaces, calculated from the phenomenological theory. We specify $\delta_{\mathrm{P}} \equiv\left(\sigma_{m}-\sigma\right) / \sigma_{\mathrm{AB}}=2$, where $\sigma_{m}=\sigma_{\mathrm{sA}-\mathrm{B}}=\sigma_{\mathrm{sB}-\mathrm{A}}$ and $\sigma=\sigma_{\mathrm{sA}-\mathrm{A}}=\sigma_{\mathrm{sB}-\mathrm{B}}$. The open symbols represent the morphology observed in Monte Carlo Simulations (Ref. 21): $\square$ denotes $\| \mid, \nabla$ denotes \|\|$_{s}-|| \mid, \bigcirc$ denotes $\mid \|_{s}, \diamond$ denotes $+[1]-|| \mid$, and $\Delta$ denotes $+[2]$. 
types of morphology have a lower free energy than the $+[m]$ morphology on the two vertical lines at $p=3$ shown in Fig. 3 (one is from $d=0.6786$ to 0.7583 , and the other is $d>1$ ). Similarly, the $+[1]-|| \mid$ morphology has a lower free energy than the $+[\mathrm{m}]$ morphology on the two vertical lines at $p=5$ shown in Fig. 3 (one is from $d=1.1953$ to 1.2902, and the other is $d>1.5$ ).

Our simulation results are also shown in Fig. 3; different open symbols represent different observed types of morphology. From Fig. 3 we can see that there is good qualitative agreement between our simulation results and theoretical predictions. Note, however, that there are some discrepancies on morphology at $(p, d)=\left(\frac{2}{3}, 1\right)$ and $(1.5,1)$.

Pereira and Williams studied the phase diagram of symmetric diblock copolymer thin films confined between patterned-neutral surfaces in Ref. 27 and 28, where they restricted their study to the case $L_{s} / L_{0}<1$, and where they only considered the morphology of perpendicular lamellae of different periods forming throughout the entire film. The "diblock-stripe" model they used is similar to ours, but their methodology artificially imposes periodic boundary conditions on the morphology with a period $n L_{s} / 2$ along the direction in which the surface is patterned (the $y$ direction in our notation). This might lead to some unexpected results. For example, in Ref. 27 and 28 it was concluded that a stripe-patterned surface can induce some "incommensurate" morphology, i.e., perpendicular lamellae of unequal spacing or even inverted bilayers, where an A-B lamella is followed directly by another $\mathrm{A}-\mathrm{B}$ lamella rather than a $\mathrm{B}-\mathrm{A}$ (each lamella consists of one layer of copolymer chains). However, we have compared under the same conditions the free energy of all the "incommensurate" morphology reported in Ref. 28 with that of the || $\mid$ morphology studied here; we have found that the || $\mid$ morphology always has a lower free energy. Note that in some cases the || $\mid$ morphology may not even be the morphology with the lowest free energy (the $\mid \|_{s}$ or the $\|\left.\right|_{s^{-}}|| \mid$morphology may be more stable).

In a recent paper, to validate the presence of the "incommensurate" morphology, Pereira and Williams studied symmetric diblock copolymer thin films confined between patterned-neutral surfaces with several hundreds of stripes by direct numerical integration of a dynamic evolution equation given by the Cahn-Hilliard formalism. ${ }^{41}$ However, since that study was restricted to one dimension and only considered the morphology of perpendicular lamellae of different periods (forming throughout the entire film), the formation of the $\left.||\right|_{s^{-}}|| \mid$morphology was excluded. This morphology could have a lower free energy than the "incommensurate" morphology under certain conditions. Furthermore, as pointed out by the authors, the sinusoidal profiles of the order parameter indicate that their results are not quantitatively valid in the strong segregation limit. ${ }^{41}$ We therefore believe that the existence of the "incommensurate" morphology remains an open question.

\section{Phase diagram for thin films confined between patterned-preferential surfaces}

In this case we set $\delta_{\mathrm{P}}=2$ and $d=2$, and vary $p$ within $\left[\frac{1}{5}, 5\right]$ and $\delta_{\mathrm{H}}$ within $[0,2]$ in the phase diagram. Note that

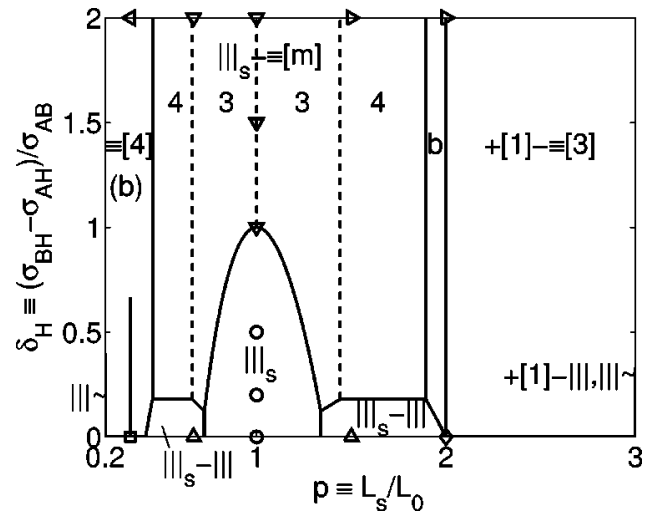

FIG. 4. Phase diagram of symmetric diblock copolymer thin films confined between patterned-preferential surfaces, calculated from the phenomenological theory. We specify $\delta_{\mathrm{P}}=2$ and $d=2$. For the $\|\left.\right|_{s^{-}} \equiv[m]$ morphology, the value of $m$ is given in the figure. The open symbols represent the morphology observed in Monte Carlo simulations (Ref. 21): $\square$ denotes $\|, \triangle$ denotes \|\|$_{s}-\| \mid, \bigcirc$ denotes \|\|$_{s}, \diamond$ denotes $+[1]-\| \mid, \triangleleft$ denotes $\equiv[4], \nabla$ denotes \|\|$_{s^{-}} \equiv[m]$, and $\triangleright$ denotes $+[1]-\equiv[3]$.

$\delta_{\mathrm{H}}=0$ corresponds to the patterned-neutral surfaces. According to our Monte Carlo simulation results, ${ }^{21}$ we discard the $+[m]$ morphology, and include the $\equiv[m]$, the \|\|$_{s^{-}} \equiv[m]$, and the $+[1]-\equiv[m-1]$ morphology for theoretical consideration.

The calculated phase diagram is shown in Fig. 4. At $\delta_{\mathrm{H}}=0$, the $\|||$ morphology coexists with the $\equiv[4]$ morphology (except at $p=1 / 5$ and $1 / 3$ ), and the $+[1]-|| \mid$ morphology coexists with the $+[1]-\equiv[3]$ morphology (except at $p$ $=3$ and 5). Note that $d=2$ for the phase diagram; similar to the case of two neutral surfaces ${ }^{36}$ since the phenomenological theory does not take hard-surface effects ${ }^{5}$ into account, it cannot predict in a unique manner the formation of perpendicular lamellae near the upper neutral surface. However, this can be compensated, in some sense, if we apply prior knowledge of the morphology obtained from our simulations to exclude the $\equiv[m]$, the \|\|$_{s^{-}} \equiv[m]$, and the $+[1]-\equiv[m$ -1 ] morphology at $\delta_{\mathrm{H}}=0$ (parallel lamellae have never been observed near a neutral surface in our Monte Carlo simulations $^{5,21}$ ).

In Fig. 4, for the \|\|$_{s^{-}} \equiv[m]$ morphology, $m=1$ with $h$ $=1.5, m=2$ with $h=1$, and $m=3$ with $h=0.5$ coexist at $p$ $=1$. Because of the "unusual" values of $q$ at $p=1 / 5,1 / 3,3$, and 5, the $\| \mid$ morphology has a lower free energy than the $\equiv[4]$ morphology on the vertical lines at $p=1 / 5$ (from $\delta_{H}$ $=0$ to $2 / 5$ ) and $1 / 3$ (from $\delta_{\mathrm{H}}=0$ to 2/3) shown in Fig. 4; the $+[1]-|| \mid$ morphology has a lower free energy than the $+[1]-\equiv[3]$ morphology on the vertical lines at $p=3$ (from $\delta_{\mathrm{H}}=0$ to $1 / 3$ ) and 5 (from $\delta_{\mathrm{H}}=0$ to $1 / 5$, not shown in Fig. $4)$; the ||| morphology coexists with the $+[1]-|| \mid$ morphology at $p=3$.

Our simulation results are also shown in Fig. 4; different open symbols represent different observed types of morphology. Again, from Fig. 4 we can see that we have good qualitative agreement with theoretical predictions. Note that there is a discrepancy on morphology at $(p, d)=(1.5,2)$.

In their study of the phase diagram of symmetric diblock copolymer thin films confined between patternedhomogeneous surfaces ${ }^{29}$ Pereira and Williams considered 


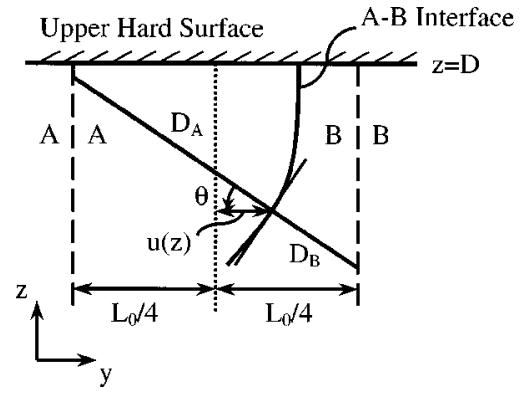

FIG. 5. Illustration of an A-B perpendicular lamella near the upper hard surface. The lower surface (not shown) locates at $z=0$. The $x$ direction is perpendicular to the page. The dotted line represents the A-B interface in the undistorted state. (Here the upper surface prefers the A blocks, i.e., $\delta_{\mathrm{H}}$ $>0$.) The two dashed lines represent the invariant interfaces between adjacent chain layers. It is assumed that chain trajectories are perpendicular to the $\mathrm{A}-\mathrm{B}$ interface and that the chains terminate at the interfaces between adjacent chain layers.

the following five types of morphology: \|\|$_{s}, \| \mid, \equiv[m]$, $\|\left.\right|_{s^{-}} \equiv[m]$ (these four types of morphology are also considered in this paper), and $|\||-\equiv[m]$ (this morphology has never been observed in our simulations and in their phase diagrams $\left.{ }^{29}\right)$. Since other types of morphology $\left(\|\|_{s^{-}} \mid \|\right.$, $+[m],+[1]-\mid \|$, and $+[1]-\equiv[m-1])$ are observed in our Monte Carlo simulations,${ }^{21}$ they should be considered in theoretical work.

\section{B. Undulation of perpendicular lamellae}

We have seen that there is qualitative agreement between the phase diagrams predicted by the phenomenological theory and our Monte Carlo simulation results for thin films confined between patterned-homogeneous surfaces. In this section, we revisit the undulation of perpendicular lamellae between two hard surfaces using the same theory, and make quantitative comparison between the theory and our simulations.

\section{Undulation between two homogeneous and preferential surfaces}

Let us first consider the case of perpendicular lamellae confined between two homogeneous, hard, flat, and preferential surfaces. As in Ref. 19, the two surfaces are located at $z=0$ and $z=D$, respectively. We assume that perpendicular lamellae of period $L_{0}$ form along the $y$ direction. Because of the surface preference, the $\mathrm{A}-\mathrm{B}$ interfaces in the perpendicular lamellae undergo a displacement $u(z)$ from the undistorted reference state, as illustrated in Fig. 5. The interfaces between adjacent chain layers, however, are assumed to be invariant. To keep the morphology of perpendicular lamellae, we specify $|u(z)|<L_{0} / 4$. Assuming $u_{i}(z)=-u_{i+1}(z)$ for two adjacent chain layers $i$ and $i+1$, as in Ref. 19, we consider one A-B lamella (consisting of one layer of copolymer chains) of unit length in the $x$ direction. Following the assumptions in Ref. 19, namely, that chain trajectories are perpendicular to the A-B interface and that chains terminate at the interfaces between adjacent lamellae, the elastic free energy of an A-B lamella per unit length in the $x$ direction can be written as [from Eq. (4)],

$$
F_{e l}=8 a \rho \frac{L_{0}}{2} \int_{0}^{D}\left(D_{\mathrm{A}}^{2}+D_{\mathrm{B}}^{2}\right) \mathrm{d} z,
$$

where:

$$
\begin{aligned}
& D_{\mathrm{A}}(z)=\frac{L_{0} / 4+u(z)}{\cos \theta(z)}, \\
& D_{\mathrm{B}}(z)=\frac{L_{0} / 4-u(z)}{\cos \theta(z)}, \\
& \theta(z)=\arctan u_{z},
\end{aligned}
$$

and where $u_{z}$ represents $\mathrm{d} u(z) / \mathrm{d} z$. Substitution of Eqs. (6) and (10) into Eq. (9) gives

$$
F_{e l}=\frac{\sigma_{\mathrm{AB}}}{2} \int_{0}^{D}\left(1+u_{z}^{2}\right)\left[1+16\left(\frac{u}{L_{0}}\right)^{2}\right] \mathrm{d} z .
$$

In this case the curvature is given by $c=u_{z z} \cdot{ }^{19,35}$ Therefore, the bending free energy of an A-B lamella per unit length in the $x$ direction is [using Eq. (6)]

$$
F_{\text {bend }}=\frac{3 \sigma_{\mathrm{AB}}}{128} \int_{0}^{D}\left(u_{z z} L_{0}\right)^{2} \mathrm{~d} z .
$$

The A-B interfacial energy of an A-B lamella per unit length in the $x$ direction is

$$
F_{A B}=\sigma_{\mathrm{AB}} \int_{0}^{D} \sqrt{1+u_{z}^{2}} \mathrm{~d} z .
$$

The surface-block interfacial energy of an A-B lamella per unit length in the $x$ direction is:

$$
F_{\text {surf }}=\left(\sigma_{\mathrm{AS}}-\sigma_{\mathrm{BS}}\right) u(0)+\left(\sigma_{\mathrm{AH}}-\sigma_{\mathrm{BH}}\right) u(D),
$$

where subscripts $\mathrm{S}$ and $\mathrm{H}$ represent the lower and upper homogeneous surfaces, respectively. The total free energy of an A-B lamella per unit length in the $x$ direction is therefore

$$
F_{T}=F_{\text {el }}+F_{\text {bend }}+F_{A B}+F_{\text {surf }} \text {. }
$$

After introducing dimensionless variables, $s=z / L_{0}, f$ $=u / L_{0}, \mathcal{F}=F_{T} /\left(\sigma_{\mathrm{AB}} L_{0}\right)$, and $\delta_{\mathrm{S}}=\left(\sigma_{\mathrm{BS}}-\sigma_{\mathrm{AS}}\right) / \sigma_{\mathrm{AB}}$, and ignoring all constants, we arrive at the following expression for the dimensionless free energy of an A-B lamella per unit length in the $x$ direction,

$$
\begin{aligned}
\mathcal{F}= & \int_{0}^{d}\left[8 f^{2}+\frac{1}{2} f_{s}^{2}+8 f^{2} f_{s}^{2}+\left(\sqrt{1+f_{s}^{2}}-1\right)+\frac{3}{128} f_{s s}^{2}\right] \mathrm{d} s \\
& -\delta_{\mathrm{S}} f(0)-\delta_{\mathrm{H}} f(d) .
\end{aligned}
$$

Note that the value of $a$ in Eq. (1) does not affect this expression for the dimensionless free energy, and therefore does not affect the corresponding Euler-Lagrange equations derived below. To obtain a linear Euler-Lagrange equation, we now assume that $f(s) \ll 1$ and that $f_{s}(s) \ll 1$, and Taylor expand the integrand in Eq. (16) to second order. We get

$$
\mathcal{F}=\int_{0}^{d}\left[8 f^{2}+f_{s}^{2}+\frac{3}{128} f_{s s}^{2}\right] \mathrm{d} s-\delta_{\mathrm{S}} f(0)-\delta_{\mathrm{H}} f(d) .
$$

Unfortunately, in Ref. 19 a factor of $\frac{1}{2}$ was apparently omitted in the right-hand side (RHS) of Eqs. (9) and (11), and a factor of $\frac{1}{4}$ was omitted in the RHS of Eq. (12); different 
coefficients were therefore obtained in this equation and the corresponding linear Euler-Lagrange equation (given below).

To minimize the free energy under the volume constraint,

$$
\int_{0}^{d} f(s) \mathrm{d} s=0,
$$

we define a functional,

$$
\Phi\left(f, f_{s}, f_{s s}\right)=\mathcal{F}+\Lambda \int_{0}^{d} f(s) \mathrm{d} s,
$$

where $\Lambda$ is a Lagrange multiplier. Minimizing $\Phi$ with respect to $f(s)$ gives us the following linear Euler-Lagrange equation:

$$
\frac{3}{64} f_{s s s s}-2 f_{s s}+16 f=-\Lambda .
$$

As in Ref. 19, the following fixed boundary conditions are employed to represent the hard surface confinement, ${ }^{42}$

$$
f_{s}(0)=f_{s}(d)=0 .
$$

The natural boundary conditions for minimizing $\Phi$ are

$$
f_{s s s}(0)=\frac{64}{3} \delta_{\mathrm{S}}, \quad f_{s s s}(d)=-\frac{64}{3} \delta_{\mathrm{H}} .
$$

The factor $\frac{64}{3}$ was omitted in these natural boundary conditions in earlier work, ${ }^{19}$ which is probably the reason why small amplitudes were reported for undulations. Our solution is

$$
\begin{aligned}
f(s)= & {\left[\exp \left(\eta_{1} s\right)-\frac{\exp \left(\eta_{1} d\right)-1}{\eta_{1} d}\right] A_{1} } \\
& +\left[\exp \left(-\eta_{1} s\right)+\frac{\exp \left(-\eta_{1} d\right)-1}{\eta_{1} d}\right] A_{2} \\
& +\left[\exp \left(\eta_{2} s\right)-\frac{\exp \left(\eta_{2} d\right)-1}{\eta_{2} d}\right] A_{3} \\
& +\left[\exp \left(-\eta_{2} s\right)+\frac{\exp \left(-\eta_{2} d\right)-1}{\eta_{2} d}\right] A_{4},
\end{aligned}
$$

where $\eta_{1}=4 \sqrt{2}, \eta_{2}=4 \sqrt{\frac{2}{3}}$, and

$$
\begin{aligned}
& A_{1}=\frac{64}{3} \frac{\delta_{\mathrm{S}}+\delta_{\mathrm{H}} \exp \left(\eta_{1} d\right)}{\eta_{1}\left(\eta_{2}^{2}-\eta_{1}^{2}\right)\left[\exp \left(2 \eta_{1} d\right)-1\right]}, \\
& A_{2}=\frac{64}{3} \frac{\delta_{\mathrm{S}} \exp \left(2 \eta_{1} d\right)+\delta_{\mathrm{H}} \exp \left(\eta_{1} d\right)}{\eta_{1}\left(\eta_{2}^{2}-\eta_{1}^{2}\right)\left[\exp \left(2 \eta_{1} d\right)-1\right]}, \\
& A_{3}=-\frac{64}{3} \frac{\delta_{\mathrm{S}}+\delta_{\mathrm{H}} \exp \left(\eta_{2} d\right)}{\eta_{2}\left(\eta_{2}^{2}-\eta_{1}^{2}\right)\left[\exp \left(2 \eta_{2} d\right)-1\right]}, \\
& A_{4}=-\frac{64}{3} \frac{\delta_{\mathrm{S}} \exp \left(2 \eta_{2} d\right)+\delta_{\mathrm{H}} \exp \left(\eta_{2} d\right)}{\eta_{2}\left(\eta_{2}^{2}-\eta_{1}^{2}\right)\left[\exp \left(2 \eta_{2} d\right)-1\right]}
\end{aligned}
$$

We recalculate the undulation of the A-B interfaces in the perpendicular lamellae, $f(s)$, for the three cases

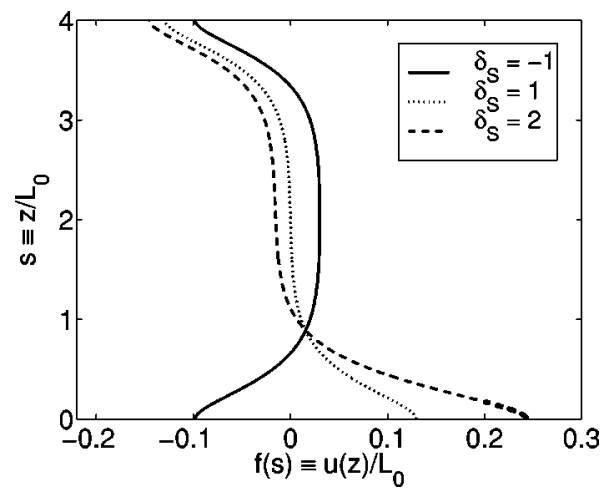

FIG. 6. Undulation of the A-B interface in perpendicular lamellae confined between two homogeneous and preferential surfaces, calculated for $d=4$ and $\delta_{\mathrm{H}}=-1$ using the fixed boundary conditions Eq. (21). For the strongly preferential surface $\left(\delta_{\mathrm{S}}=2\right)$, the thick dashed line (near the lower surface) represents the region where the solution does not satisfy the hard surface confinement expressed in Ref. 42. Our Monte Carlo simulations show that parallel lamellae actually form in all these cases due to the relatively strong surface preference.

( $\delta_{\mathrm{S}}=-1,1$, and $2 ; \delta_{\mathrm{H}}=-1$ and $d=4$ in all the cases) studied in Ref. 19. Our results are shown in Fig. 6. We can see that near these surfaces with relatively strong preference for one of the two blocks, if perpendicular lamellae could form throughout the entire film, the amplitude of the undulation, defined as $A=|f(d)|$, would be on the order of $10^{-1}$, one order of magnitude larger than reported in the literature. ${ }^{19}$ Note, however, that our Monte Carlo simulations show that, due to the relatively strong surface preference, parallel lamellae actually occur in all three cases. The number of chain layers in the parallel lamellae is either 8 (in the case of $\delta_{\mathrm{S}}=-1$ ), or 9 (in the cases of $\delta_{\mathrm{S}}=1$ and 2). Figure 7 shows a representative configuration of the system from a simulation for the case $\delta_{\mathrm{S}}=1$.

For a direct comparison to our simulation data, we calculate using the above solution the undulation of the A-B interfaces in perpendicular lamellae confined between two weakly preferential surfaces, i.e., $\delta_{\mathrm{S}}=\delta_{\mathrm{H}}=-0.5$ (symmetric surfaces where $d=1.5$ ) and $\delta_{\mathrm{S}}=-\delta_{\mathrm{H}}=-0.5$ (antisymmetric surfaces where $d=2$ ). Perpendicular lamellae were observed in Monte Carlo simulations for these two cases. ${ }^{5}$ From the
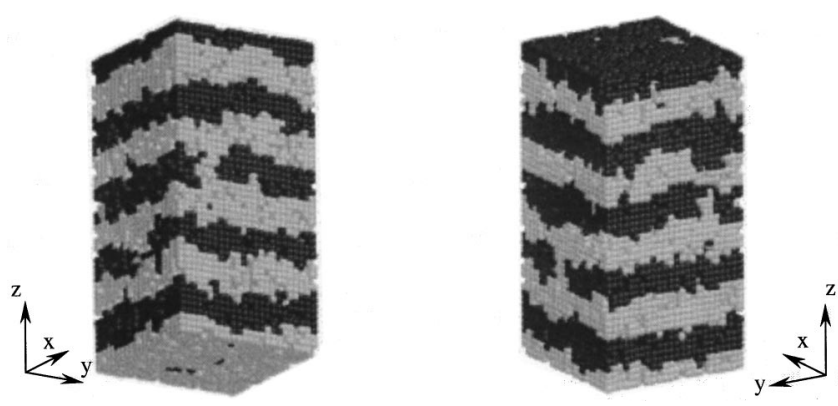

FIG. 7. Representative configuration of the system from a Monte Carlo simulation for two homogeneous and preferential surfaces with $\delta_{\mathrm{S}}=1(d$ $=4$ and $\delta_{\mathrm{H}}=-1$ ). The six surfaces of the simulation box are shown in the figure. Light regions represent A blocks and dark regions represent B blocks. Due to the relatively strong surface preference, parallel lamellae with nine chain layers form within the confined film. 


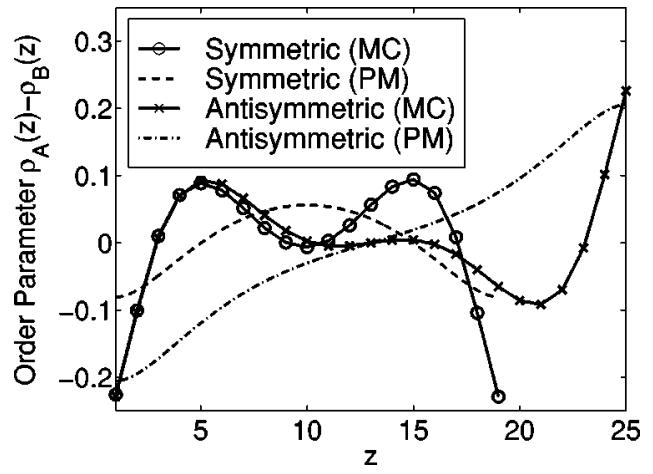

FIG. 8. Order parameter profiles along the $z$ direction, calculated from Monte Carlo simulations (MC) and the phenomenological theory (PM), of perpendicular lamellae confined between symmetric and weakly preferential surfaces $\left(d=1.5, \delta_{\mathrm{S}}=\delta_{\mathrm{H}}=-0.5\right)$, and antisymmetric and weakly preferential surfaces $\left(d=2, \delta_{\mathrm{S}}=-\delta_{\mathrm{H}}=-0.5\right)$.

assumption $u_{i}(z)=-u_{i+1}(z)$ we get a simple relation to convert $f(s)$ to the order parameter profile along the $z$ direction, $\rho_{\mathrm{A}}(z)-\rho_{\mathrm{B}}(z),^{5}$

$$
\rho_{\mathrm{A}}(z)-\rho_{\mathrm{B}}(z)=4 \rho f\left(z / L_{0}\right),
$$

where $\rho$ is the total percentage of lattice sites occupied by A and $\mathrm{B}$ segments in the system, taken from the simulation data. The results are shown in Fig. 8. We can see that the phenomenological theory predicts the correct order of magnitude of the amplitudes of the undulations. However, it is unable to describe the peaks in the order parameter profiles near the surfaces shown by simulations (at $z=5$ and 15 in the case of symmetric surfaces, and at $z=5$ and 21 in the case of antisymmetric surfaces, as shown in Fig. 8). Similar peaks were also observed in Monte Carlo simulations in the framework of the bond fluctuation model ${ }^{18}$ and selfconsistent field calculations. ${ }^{10,11,17,18}$

We note here that Eq. (16) can also be minimized without assuming $f(s) \ll 1$ and $f_{s}(s) \ll 1$. This gives rise to the following nonlinear Euler-Lagrange equation:

$$
\frac{3}{64} f_{s s s s}-\left[\left(1+f_{s}^{2}\right)^{-(3 / 2)}+1+16 f^{2}\right] f_{s s}+16\left(1-f_{s}^{2}\right) f=-\Lambda \text {. }
$$

The boundary conditions are unchanged. Equation (26) can be solved numerically [together with the volume constraint Eq. (18)]. We find that for the above case (symmetric and weakly preferential surfaces), where perpendicular lamellae were observed in Monte Carlo simulations, the numerical solution is almost identical to the analytical solution of the linear Euler-Lagrange equation. Since $|f(s)|<0.03$ and $\left|f_{s}(s)\right|<0.1$ in that case, the two solutions are almost undistinguishable in Fig. 8. This confirms that the Taylor expansion is valid for small $f(s)$ and $f_{s}(s)$.

\section{Undulation between patterned-preferential surfaces}

In this case, the surface at $z=0$ is stripe-patterned along the $y$ direction, as shown in Fig. 1. All the stripes have the same width $L_{s} / 2$, and alternatively prefer A and B blocks. The upper surface at $z=D$ is homogeneous and preferential to one of the two blocks. Pereira and Williams also studied the undulation of perpendicular lamellae for this surface configuration. ${ }^{37}$ These authors assumed that perpendicular lamellae complying with the lower surface pattern form throughout the entire film. The surface-block interfacial energy for the lower patterned surface was therefore 0 . The assumptions for undulation of Sec. III B 1 were adopted. That is, the $\mathrm{A}-\mathrm{B}$ interfaces undergo a displacement $u(z)$ from the undistorted reference state, while the interfaces between adjacent lamellae are assumed to be invariant; for two adjacent lamellae $i$ and $i+1, u_{i}(z)=-u_{i+1}(z)$; chain trajectories are perpendicular to the A-B interface, and chains terminate at the interfaces between adjacent lamellae. ${ }^{37}$

In this case, the elastic free energy of an A-B lamella per unit length in the $x$ direction can be obtained from Eqs. (9) and (10) by replacing $L_{0}$ with $L_{s}$,

$$
F_{e l}=8 a \rho \frac{L_{s}}{2} \int_{0}^{D}\left(D_{\mathrm{A}}^{2}+D_{\mathrm{B}}^{2}\right) \mathrm{d} z,
$$

where

$$
\begin{aligned}
& D_{\mathrm{A}}(z)=\frac{L_{s} / 4+u(z)}{\cos \theta(z)}, \\
& D_{\mathrm{B}}(z)=\frac{L_{s} / 4-u(z)}{\cos \theta(z)}, \\
& \theta(z)=\arctan u_{z} .
\end{aligned}
$$

The bending free energy of an A-B lamella per unit length in the $x$ direction, Eq. (12), becomes

$$
F_{\text {bend }}=\frac{3 \sigma_{\mathrm{AB}} L_{s}^{5}}{128 L_{0}^{5}} \int_{0}^{D}\left(u_{z z} L_{0}\right)^{2} \mathrm{~d} z
$$

The A-B interfacial energy of an A-B lamella per unit length in the $x$ direction, Eq. (13), remains unchanged. But the surface-block interfacial energy of an A-B lamella per unit length in the $x$ direction, Eq. (14), becomes:

$$
F_{\text {surf }}=\left(\sigma_{\mathrm{AH}}-\sigma_{\mathrm{BH}}\right) u(D) .
$$

By using dimensionless variables and ignoring all constants, we get the dimensionless free energy of an A-B lamella per unit length in the $x$ direction,

$$
\begin{aligned}
\mathcal{F}= & \int_{0}^{d}\left[8 p f^{2}+\frac{p^{3}}{2} f_{s}^{2}+8 p f^{2} f_{s}^{2}+\left(\sqrt{1+f_{s}^{2}}-1\right)\right. \\
& \left.+\frac{3 p^{5}}{128} f_{s s}^{2}\right] \mathrm{d} s-\delta_{\mathrm{H}} f(d) .
\end{aligned}
$$

Again, we can see that the value of $a$ in Eq. (1) does not affect this expression for the dimensionless free energy, and therefore does not affect the Euler-Lagrange equation derived below. Assuming $f(s) \ll 1$ and $f_{s}(s) \ll 1$, and Taylor expanding the integrand in Eq. (31) to second order, we get

$$
\mathcal{F}=\int_{0}^{d}\left(8 p f^{2}+\frac{p^{3}+1}{2} f_{s}^{2}+\frac{3 p^{5}}{128} f_{s s}^{2}\right) \mathrm{d} s-\delta_{\mathrm{H}} f(d) .
$$

In Ref. 37 a factor of $\frac{1}{2}$ was omitted in the right-hand side (RHS) of Eq. (27), and a factor of $\left[1 /\left(4 L_{0}^{2}\right)\right]$ was omitted in 


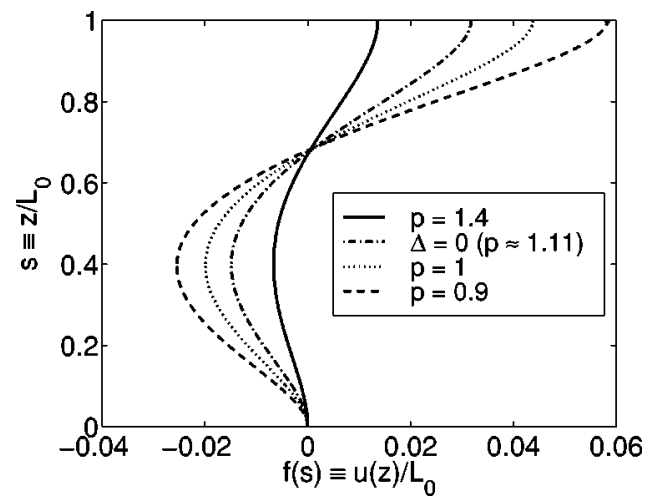

FIG. 9. Undulation of the A-B interface in perpendicular lamellae confined between patterned-preferential surfaces, calculated for $\delta_{\mathrm{H}}=1$ and $d=1$ using the fixed boundary conditions Eq. (21). Our Monte Carlo simulations for the cases of $p=1$ and $p=1.5\left(\delta_{\mathrm{H}}=1\right.$ and $\left.d=1\right)$ show that parallel lamellae actually form near the upper preferential surface in both cases due to the relatively strong surface preference.

the RHS of Eq. (29); different coefficients were therefore obtained in this equation and the corresponding EulerLagrange equation (given below).

Following a procedure analogous to that presented in Sec. III B 1, we get the following Euler-Lagrange equation:

$$
\frac{3 p^{5}}{64} f_{s s s s}-\left(p^{3}+1\right) f_{s s}+16 p f=-\Lambda \text {. }
$$

The fixed boundary conditions are $f(0)=0$ and $f_{s}(0)$ $=f_{s}(d)=0$. The natural boundary condition is $f_{s s s}(d)$ $=-\left[64 /\left(3 p^{5}\right)\right] \delta_{\mathrm{H}}$. The factor $64 /\left(3 p^{5}\right)$ in this natural boundary condition was omitted in earlier work, ${ }^{37}$ where small amplitudes were reported for undulations. See Appendix A for our solution.

To compare with Ref. 37, we calculate the undulation of the A-B interfaces in perpendicular lamellae, $f(s)$, for four different values of $p$ at $\delta_{\mathrm{H}}=1$ and $d=1$ (assuming that perpendicular lamellae form in these cases); the results are shown in Fig. 9. We can see that if perpendicular lamellae could form throughout the entire film in these cases, the amplitude of the undulation would be on the order of $10^{-2}$, instead of the order of $10^{-3}$ reported in the literature. ${ }^{37}$ Furthermore, the amplitude would decrease as $p$ increases; this is contrary to earlier reports. ${ }^{37}$ Note, however, that our Monte Carlo simulations for the cases of $p=1$ and $p=1.5$, with $\delta_{\mathrm{H}}=1$ and $d=1$, show that parallel lamellae actually form near the upper preferential surface due to the relatively strong surface preference. ${ }^{21}$ Figure 10 shows a representative configuration of the system from a simulation for the case $p=1$.

Our Monte Carlo simulations ${ }^{21}$ have shown that when $p$ deviates far from unity, perpendicular lamellae complying with the lower surface pattern cannot be obtained, even near the patterned surface. For a small value of $p$ (e.g., $\left.\frac{1}{3}\right)$ the lower surface pattern would be ignored, while for a large value of $p$ (e.g., 2) chains near the patterned surface would change their orientation to be perpendicular to the surface, thereby leading to one layer of checkerboard morphology. On the other hand, our Monte Carlo simulations also indicate that parallel lamellae actually form near the upper preferen-
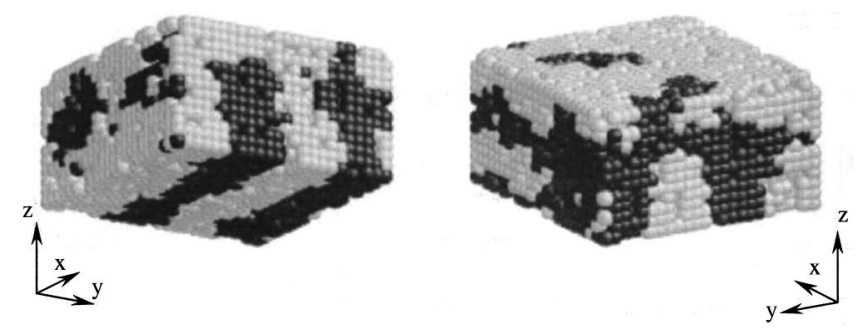

FIG. 10. Representative configuration of the system from a Monte Carlo simulation for patterned-preferential surfaces with $p=1 \quad\left(\delta_{\mathrm{H}}=1\right.$ and $d$ $=1$ ). The six surfaces of the simulation box are shown in the figure. Light regions represent A blocks and dark regions represent B blocks. Perpendicular lamellae complying with the surface pattern form near the lower patterned surface, and one layer of parallel lamellae forms near the upper surface due to the relatively strong surface preference.

tial surface when the surface preference is relatively strong. Even for the case of a neutral surface on the top (this case is discussed in the following section) and $p$ not far from unity (e.g., $\frac{2}{3}$ or 1.5 ), where a perpendicular structure is observed in the confined films, the structure actually consists of perpendicular lamellae complying with the surface pattern near the lower patterned surface, and perpendicular lamellae of period $L_{0}$ near the upper neutral surface. ${ }^{21}$ Therefore, the basic assumption adopted in Ref. 37 and 38, that perpendicular lamellae complying with the lower surface pattern form throughout the entire film, is valid only when $p$ is very close to unity and the upper homogeneous surface is either neutral or weakly preferential.

To compare the prediction of the phenomenological theory with Monte Carlo simulations when perpendicular lamellae complying with the lower surface pattern actually occur throughout the entire film, we simulate the cases of weakly preferential surface on the top, i.e., $\delta_{\mathrm{H}}=0.5$ and 0.25 , with $p=1$ and $d=1$. Figure 11 shows a representative configuration of the system from a simulation for the case $\delta_{\mathrm{H}}$ $=0.25$. Equation $(25)$ is used to convert the predicted undulation to the order parameter profile. The results are shown in Fig. 12. We can see that the amplitude of the undulation obtained from simulations is larger than that predicted by the theory. More importantly, as in Fig. 8 (the cases of perpendicular lamellae confined between two homogeneous and preferential surfaces), the phenomenological theory is again unable to describe the peaks (at $z=3$ in Fig. 12) shown by Monte Carlo simulations.
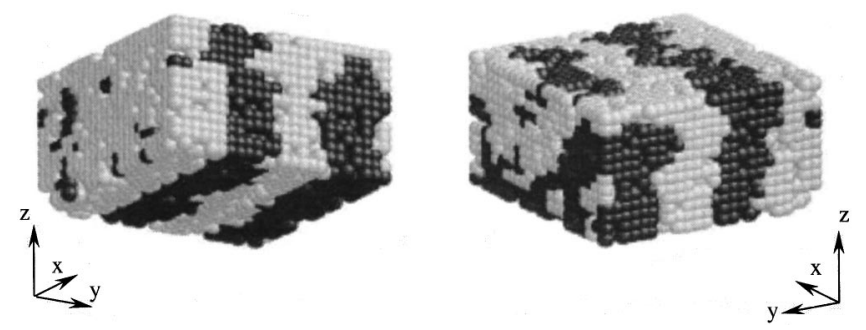

FIG. 11. Representative configuration of the system from a Monte Carlo simulation for patterned-preferential surfaces with $\delta_{\mathrm{H}}=0.25(p=1$ and $d$ $=1$ ). The six surfaces of the simulation box are shown in the figure. Light regions represent A blocks and dark regions represent B blocks. Perpendicular lamellae complying with the lower surface pattern form throughout the entire film. 


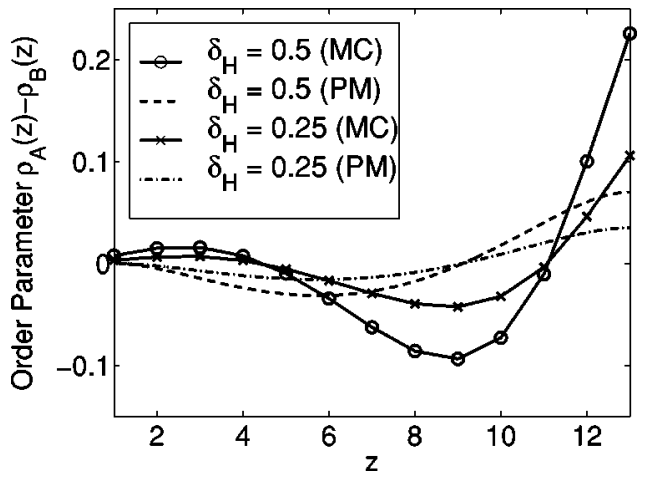

FIG. 12. Order parameter profiles along the $z$ direction, calculated from Monte Carlo simulations (MC) and the phenomenological theory (PM), for perpendicular lamellae confined between a lower stripe-patterned and an upper weakly preferential surface $(p=1$ and $d=1)$.

\section{Undulation between patterned-neutral surfaces}

In this case the lower stripe-patterned surface has a period $L_{s} \geqslant L_{0}$, and the upper surface at $z=D$ is neutral (refer to Fig. 1). In a separate paper, ${ }^{38}$ Pereira and Williams applied a similar theory to study the undulation of perpendicular lamellae confined between patterned-neutral surfaces. They assumed that perpendicular lamellae complying with the lower surface pattern form throughout the entire film. The surface-block interfacial energy is therefore $F_{\text {surf }}=0$. Different from the above two cases, when $L_{s}>L_{0}$, these authors assumed that the $\mathrm{A}-\mathrm{B}$ interfaces undergo a displacement $u(x, z)$ from the undistorted reference state to relieve the imposed strain $p$, and that the interfaces between adjacent lamellae undergo the same displacement as the A-B interfaces. The volume constraint is therefore satisfied automatically. If it is assumed that all the second derivatives of $u$ with respect to $x$ and $z$ (i.e., $u_{x x}, u_{x z}$, and $u_{z z}$ ) are small, such an undulation of the perpendicular lamellae reduces the effective lamellar spacing (perpendicular to the $\mathrm{A}-\mathrm{B}$ interfaces) approximately to $L=L_{s} / \sqrt{\left(1+u_{x}^{2}\right)\left(1+u_{z}^{2}\right)}$, and $D_{\mathrm{A}}=D_{\mathrm{B}}$ $=L / 4$ (chain trajectories are again assumed to be perpendicular to the A-B interfaces). Therefore, the elastic free energy of an A-B lamella (from $x=-\infty$ to $\infty$ ) is

$$
\widetilde{F}_{e l}=\frac{\sigma_{\mathrm{AB}} L_{s}^{3}}{2 L_{0}^{3}} \int_{-\infty}^{\infty} \int_{0}^{D} \frac{1}{\left(1+u_{x}^{2}\right)\left(1+u_{z}^{2}\right)} \mathrm{d} z \mathrm{~d} x .
$$

In this case, the curvature is $c=u_{x x}+u_{z z} \cdot{ }^{35,38}$ Thus, the bending free energy of an A-B lamella (from $x=-\infty$ to $\infty$ ) is

$$
\widetilde{F}_{\text {bend }}=\frac{3 \sigma_{\mathrm{AB}} L_{s}^{5}}{128 L_{0}^{5}} \int_{-\infty}^{\infty} \int_{0}^{D}\left[\frac{\left(u_{x x}+u_{z z}\right) L_{0}}{\left(1+u_{x}^{2}\right)\left(1+u_{z}^{2}\right)}\right]^{2} \mathrm{~d} z \mathrm{~d} x .
$$

The A-B interfacial energy of an A-B lamella (from $x=-\infty$ to $\infty)$ is

$$
\widetilde{F}_{A B}=\sigma_{\mathrm{AB}} \int_{-\infty}^{\infty} \int_{0}^{D} \sqrt{\left(1+u_{x}^{2}\right)\left(1+u_{z}^{2}\right)} \mathrm{d} z \mathrm{~d} x .
$$

As in Ref. 38, by adding all of the terms above, assuming $u_{x} \ll 1$ and $u_{z} \ll 1$ and Taylor expanding the integrand to sec- ond order, and ignoring all constants, the free energy of an A-B lamella (from $x=-\infty$ to $\infty$ ) can be written as

$$
\begin{aligned}
\widetilde{F}_{T}= & \frac{3 \sigma_{\mathrm{AB}}}{2} \int_{-\infty}^{\infty} \int_{0}^{D}\left[\frac{p^{5}}{64} L_{0}^{2}\left(u_{x x}+u_{z z}\right)^{2}\right. \\
& \left.-\frac{p^{3}-1}{3}\left(u_{x}^{2}+u_{z}^{2}\right)\right] \mathrm{d} z \mathrm{~d} x .
\end{aligned}
$$

By making the ansatz that $u(x, z)=\cos \left(\xi x / L_{0}\right) g(z)$, where $g(z)$ is an unknown function of $z$, then integrating over one period along the $x$ direction, Pereira and Williams ${ }^{38}$ derived the following expression for the free energy of an A-B lamella per unit length in the $x$ direction,

$$
F_{T}=\frac{3 p^{5} \sigma_{\mathrm{AB}}}{256 L_{0}} \int_{0}^{d}\left[\left(g_{s s}-\xi^{2} g\right)^{2}-\omega^{2}\left(\xi^{2} g^{2}+g_{s}^{2}\right)\right] \mathrm{d} s,
$$

where $\omega=\sqrt{64\left(p^{3}-1\right) / 3 p^{5}}$. If $\xi=0$, that is, if the problem is reduced to two dimensions in the $y-z$ plane and $u(x, z)$ $=g(z)$, then the free energy of an A-B lamella per unit length in the $x$ direction becomes

$$
F_{T}=\frac{3 p^{5} \sigma_{\mathrm{AB}}}{128 L_{0}} \int_{0}^{d}\left[g_{s s}^{2}-\omega^{2} g_{s}^{2}\right] \mathrm{d} s .
$$

Although Eq. (39) differs from Eq. (38) by a factor of 2, they have the same Euler-Lagrange equation,

$$
g_{s s s s}-\left(2 \xi^{2}-\omega^{2}\right) g_{s s}+\left(\xi^{4}-\omega^{2} \xi^{2}\right) g=0 .
$$

The fixed boundary conditions are $g(0)=0$ and $g_{s}(0)$ $=g_{s}(d)=0$. The natural boundary condition is $g_{s s s}(d)=0$.

So far we have basically followed the same procedure and obtained the same Euler-Lagrange equation and boundary conditions as in Ref. 38. However, our solution (see Appendix B) is different from that in Ref. 38. Solving the Euler-Lagrange equation subject to the above boundary conditions shows that the free energy given by Eq. (38) [or Eq. (39) when $\xi=0$ ] either has a minimum value of 0 [which means that the undulation of the A-B interfaces (if any) cannot decrease the free energy of the system], or does not have a minimum at all. This is due to the omission of higher order terms in the free energy Taylor expansion, or due to the strict boundary condition $g(0)=0$. If we allow for a nonzero value of $g(0)$ at the patterned surface, and use a natural boundary condition at $s=0$ instead, some undulations might exist that are more stable than the undistorted state. The study of such cases, however, is beyond the scope of this paper.

In what follows we partially address this problem by reducing it to two dimensions and Taylor expanding the bending free energy given in Eq. (35) to second order. By using dimensionless variables and ignoring all constants, we get the dimensionless free energy of an A-B lamella per unit length in the $x$ direction,

$$
\mathcal{F}=\int_{0}^{d}\left[\frac{p^{3}}{2}\left(\frac{1}{1+f_{s}^{2}}-1\right)+\left(\sqrt{1+f_{s}^{2}}-1\right)+\frac{3 p^{5}}{128} f_{s s}^{2}\right] \mathrm{d} s .
$$

The corresponding Euler-Lagrange equation is 


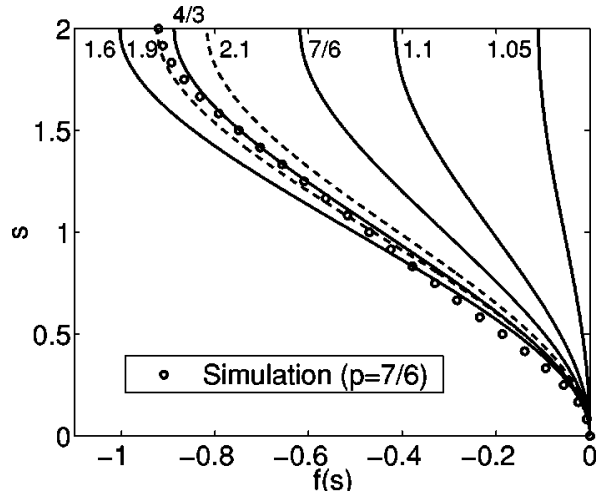

FIG. 13. Undulation of perpendicular lamellae confined between patternedneutral surfaces, calculated by solving Eq. (42) for $d=2$. The value of $p$ is given for each curve in the figure. Symmetric solutions of $f(s)>0$ are not shown in the figure. The open circles represent the undulation of perpendicular lamellae observed in a Monte Carlo simulation for $p=7 / 6$ and $d$ $=2$.

$$
\frac{3 p^{5}}{64} f_{s s s}-\frac{f_{s s}}{\left(1+f_{s}^{2}\right)^{3 / 2}}+\frac{p^{3} f_{s s}\left(1-3 f_{s}^{2}\right)}{\left(1+f_{s}^{2}\right)^{3}}=0 .
$$

The fixed boundary conditions are $f(0)=0$ and $f_{s}(0)$ $=f_{s}(d)=0$. The natural boundary condition is $f_{s s s}(d)=0$. This differential equation can be solved numerically for given $p$ and $d$. Figure 13 shows the results for $d=2$ and different values of $p$; we can see that the amplitude of the undulation has a maximum (around $p=1.6$ ) when $p$ varies. Figure 14 shows the dimensionless free energies of the undistorted perpendicular lamellae $\mid \|_{s}$ (given in Table II), the undulated perpendicular lamellae [given by $p^{2}+2 / p$ $+2 \mathcal{F} /(p d)]$, and the $\|\left.\right|_{s^{-}}|| \mid$morphology (given in Table II) for $d=2$ and $\delta_{\mathrm{H}}=0$ calculated from the phenomenological theory. We can see that the undulation of perpendicular lamellae that we obtain at this level of approximation does decrease the free energy below the undistorted state. On the other hand, the $\|\left.\right|_{s}-|| \mid$ morphology has a lower free energy than the undulated perpendicular lamellae when $p>1.4$ (for $d=2$ ); this explains why we have not observed undulated perpendicular lamellae in our simulations for thin films confined between patterned-neutral surfaces with $p \geqslant 1.5^{21}$

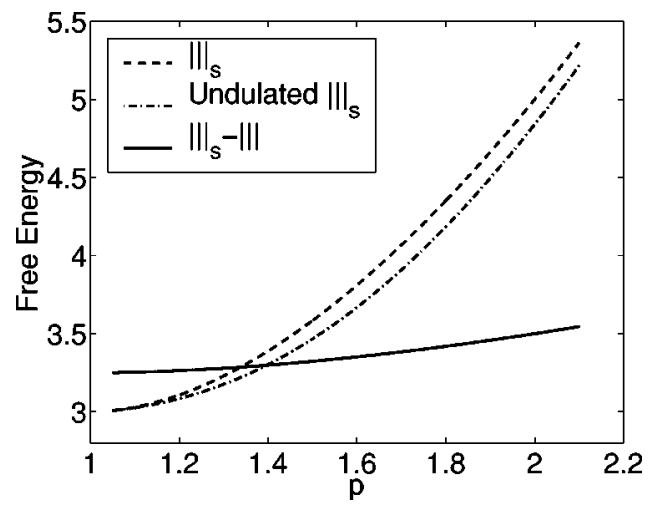

FIG. 14. Comparison of the dimensionless free energies of the undistorted perpendicular lamellae \|\|$_{s}$, undulated perpendicular lamellae, and the $\left|\|_{s}-\right|||$ morphology confined between patterned-neutral surfaces with $d=2$, calculated using the phenomenological theory.
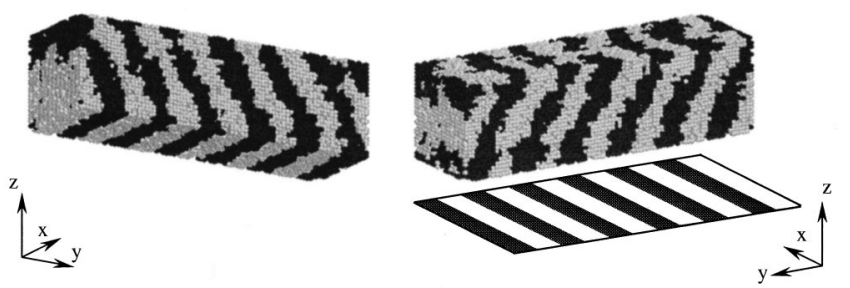

FIG. 15. Representative configuration of the system from a Monte Carlo simulation for patterned-neutral surfaces with $p=7 / 6$ and $d=2$. The six surfaces of the simulation box are shown in the figure. Light regions represent A blocks and dark regions represent B blocks. Undulated perpendicular lamellae are observed in this simulation. The right-hand side of the figure also displays the lower patterned surface, where light regions represent sA stripes and dark regions represent $\mathrm{sB}$ stripes.

Interestingly, when $p$ is close to unity, the undulated perpendicular lamellae are indeed observed in our Monte Carlo simulations for thin films confined between patternedneutral surfaces. Figure 15 shows a representative configuration of the system from a simulation for the case $d=2$ and $p=7 / 6$. From the figure we can see that the lamellae in the vicinity of the lower patterned surface comply with the surface pattern, and that the undulation of the perpendicular lamellae occurs in the $y-z$ plane, as assumed in our numerical solution. The undulation of the A-B interfaces observed in the simulation is shown in Fig. 13 by open circles. We can see that the observed undulation has a shape similar to that predicted by the phenomenological theory, but has an amplitude about $50 \%$ larger. The tilted lamellae found by Petera and Muthukumar ${ }^{26}$ in their two-dimensional self-consistent field calculations, which have an angle of $\arcsin (1 / p)$ with respect to the surface, would give an amplitude of about 1.2 under the same surface configuration. Note that the $\left.||\right|_{s^{-}}|| \mid$ morphology is also observed in some other runs in our simulations under the same conditions, with the only difference being the random number generator seeds. This may be due to the large energy barrier between the undulated perpendicular lamellae and the $\|\left.\right|_{s^{-}}|| \mid$morphology that cannot be overcome by our system in a finite simulation run.

\section{CONCLUSIONS}

We have investigated the ability of a simple phenomenological theory to describe the behavior of symmetric diblock copolymer thin films confined between two hard surfaces. Prior knowledge of the morphology in thin films is crucial for applying this theory to predict the phase diagram of such systems. Taking advantage of our observations from Monte Carlo simulations, ${ }^{21}$ we have used the theory to construct phase diagrams for thin films confined between patternedhomogeneous surfaces, and obtained good qualitative agreement with the simulations. In view of the simplicity of the theory, such agreement is both surprising and satisfactory. The phenomenological theory does not take hard-surface effects $^{5}$ into account, and is therefore unable to distinguish between the unfrustrated parallel and perpendicular lamellae near neutral surfaces. This, however, can be compensated by applying prior knowledge of the morphology obtained from Monte Carlo simulations. 
For applications to nanofabrication, our objective is to produce long-range ordered (over microns) perpendicular lamellae. This work shows that the region of parameter space in which such morphology can be found is fairly small. Two conditions are essential for obtaining this desirable morphology: a lower stripe-patterned surface with the surface pattern period $L_{s}$ comparable to the bulk lamellar period $L_{0}$, and an upper neutral or weakly preferential surface.

Following the methods of Pereira and Williams, ${ }^{19,37,38}$ we have also revisited the undulation of perpendicular lamellae between two hard surfaces. For the cases of two homogeneous (preferential) surfaces and patterned-preferential surfaces, our calculations using the phenomenological theory indicate that the amplitudes of the undulations are of the same order of magnitude as those observed in our Monte Carlo simulations, and are one order of magnitude larger than reported in the literature. ${ }^{19,37}$ These undulations could have adverse effects for lithographic applications. Unfortunately, the theory is unable to capture the shape of the undulations.

For the case of patterned-neutral surfaces, we find that a second-order expansion of the free energy ${ }^{38}$ is unable to yield the undulations that would stabilize the perpendicular lamellar morphology. In this work, we have addressed this problem in an approximate manner, and obtained undulations that can lower the free energy of the perpendicular lamellae. Such undulations are consistent with results of Monte Carlo simulations.

Our Monte Carlo simulations have provided us with valuable insights regarding the morphology and the molecular structure of the systems of interest. The simulation results have served as a guide to propose plausible solutions for theoretical calculations. Based on these results, we are currently pursuing a more refined self-consistent field theory treatment for diblock copolymer thin films confined between patterned-homogeneous surfaces.

\section{ACKNOWLEDGMENTS}

We are grateful for helpful discussions with Dr. G. G. Pereira. Financial support for this work was provided by the Semiconductor Research Corporation through Contract No. 99-LP-452 and by the NSF CTS-9703207 and CTS9901430.

\section{APPENDIX A}

Solving the characteristic equation of the homogeneous ordinary differential equation to Eq. (33) gives us four roots $\eta_{i}(i=1,2,3,4)$,

$$
\eta_{i}^{2}=\frac{32\left(p^{3}+1 \pm \sqrt{\Delta}\right)}{3 p^{5}},
$$

where $\Delta=-2 p^{6}+2 p^{3}+1$. Note that $p>0$ and $\Delta<\left(p^{3}\right.$ $+1)^{2}$. If $\Delta>0$, i.e., $0<p<[(1+\sqrt{3}) / 2]^{1 / 3}$, the general solution to Eq. (33) is

$$
\begin{aligned}
f(s)= & A_{1} \cosh \left(\eta_{1} s\right)+A_{2} \sinh \left(\eta_{1} s\right)+A_{3} \cosh \left(\eta_{2} s\right) \\
& +A_{4} \sinh \left(\eta_{2} s\right)-\frac{\Lambda}{16 p},
\end{aligned}
$$

where

$$
\eta_{1,2}=\sqrt{\frac{32\left(p^{3}+1 \pm \sqrt{\Delta}\right)}{3 p^{5}}} .
$$

$A_{1}, A_{2}, A_{3}$, and $A_{4}$ are constants to be solved from the boundary conditions. If $\Delta=0$, i.e., $p=[(1+\sqrt{3}) / 2]^{1 / 3}$, the general solution to Eq. (33) is

$$
\begin{aligned}
f(s)= & A_{1} \exp (\eta s)+A_{2} s \exp (\eta s)+A_{3} \exp (-\eta s) \\
& +A_{4} s \exp (-\eta s)-\frac{\Lambda}{16 p},
\end{aligned}
$$

where

$$
\eta=\sqrt{\frac{32\left(p^{3}+1\right)}{3 p^{5}}} .
$$

If $\Delta<0$, i.e., $p>[(1+\sqrt{3}) / 2]^{1 / 3}$, the general solution to Eq. (33) is

$$
\begin{aligned}
f(s)= & A_{1} \exp (\alpha s) \cos (\beta s)+A_{2} \exp (\alpha s) \sin (\beta s) \\
& +A_{3} \exp (-\alpha s) \cos (\beta s)+A_{4} \exp (-\alpha s) \sin (\beta s) \\
& -\frac{\Lambda}{16 p},
\end{aligned}
$$

where

$$
\alpha=4 \sqrt{\frac{(1+\sqrt{3}) p^{3}+1}{3 p^{5}}}, \quad \beta=\frac{16 \sqrt{-\Delta}}{3 p^{5} \alpha} .
$$

Similar to Sec. III B 1, from the volume constraint Eq. (18) and the four boundary conditions we can solve for the $\Lambda$ and the $A_{i}(i=1,2,3,4)$ in each case. The resultant expressions are too complicated to be listed here (they were obtained by Mathematica).

\section{APPENDIX B}

From the ansatz that $u(x, z)=\cos \left(\xi x / L_{0}\right) g(z)$ we can restrict ourselves to $\xi \geqslant 0$, without loss of generality. We are interested in nontrivial solutions for the case of $\omega>0$ (i.e., $p>1)$ and $d>0$.

Let us consider the case $\xi>0$ first. Solving the characteristic equation to Eq. (40) gives us $\eta_{1,2}^{2}=\xi^{2}$ and $\eta_{3,4}^{2}=\xi^{2}$ $-\omega^{2}$, where $\eta_{1}, \eta_{2}, \eta_{3}$, and $\eta_{4}$ are roots of the characteristic equation. If $\xi^{2}-\omega^{2}>0$, the general solution to Eq. (40) is

$$
\begin{aligned}
g(s)= & A_{1} \cosh (\xi s)+A_{2} \sinh (\xi s)+A_{3} \cosh (\zeta s) \\
& +A_{4} \sinh (\zeta s),
\end{aligned}
$$

where $\zeta=\sqrt{\xi^{2}-\omega^{2}}>0 ; A_{1}, A_{2}, A_{3}$, and $A_{4}$ are constants. Substituting Eq. (B1) into the boundary conditions gives us a system of four linear equations for the $A_{i}(i=1,2,3,4)$ with the right-hand side being all zeros. Therefore the nontrivial solution exists only if the determinant of coefficients of all the $A_{i}(i=1,2,3,4)$ on the left-hand side is zero. This gives us

$$
\xi \tanh (\xi d)=\zeta \tanh (\zeta d) .
$$


Since $\xi>\zeta>0$ and $d>0$, Eq. (B2) cannot be satisfied for any given strain $p>1$. Thus only the trivial solution $g(s)=0$ exists in this case.

If $\xi^{2}-\omega^{2}=0$, the general solution to Eq. (40) is

$$
g(s)=A_{1} \cosh (\xi s)+A_{2} \sinh (\xi s)+A_{3}+A_{4} s .
$$

Similarly, the condition for a nontrivial solution is

$$
\sinh (\xi d)=0,
$$

which obviously cannot be satisfied by any $\xi>0$ and $d>0$. Thus only the trivial solution $g(s)=0$ exists in this case.

If $\xi^{2}-\omega^{2}<0$, the general solution to Eq. (40) is

$$
\begin{aligned}
g(s)= & A_{1} \cosh (\xi s)+A_{2} \sinh (\xi s)+A_{3} \cos (\zeta s) \\
& +A_{4} \sin (\zeta s),
\end{aligned}
$$

where $\zeta=\sqrt{\omega^{2}-\xi^{2}}>0$. Similarly, the condition to obtain a nontrivial solution is

$$
\xi \cos (\zeta d) \sinh (\xi d)=-\zeta \sin (\zeta d) \cosh (\xi d) .
$$

Under this condition, the solution is

$$
g(s)=A_{1} \psi(\omega, d, s),
$$

where $A_{1}$ is an arbitrary constant, and

$$
\begin{aligned}
\psi(\omega, d, s)= & \cosh (\xi s)-\frac{\xi \sinh (\xi d)+\zeta \sin (\zeta d)}{\xi \cosh (\xi d)-\xi \cos (\zeta d)} \sinh (\xi s) \\
& -\cos (\zeta s)+\frac{\xi \sinh (\xi d)+\zeta \sin (\zeta d)}{\zeta \cosh (\xi d)-\zeta \cos (\zeta d)} \sin (\zeta s) .
\end{aligned}
$$

Note that, since $\zeta=\sqrt{\omega^{2}-\xi^{2}}$, Eq. (B6) gives $\xi$ as a function of $\omega$ and $d$ only. Substituting Eq. (B7) into Eq. (38), we can get the free energy of an undulated A-B lamella $F_{T}$ $=A_{1}^{2} \Psi(\omega, d)$, where

$$
\Psi=\frac{3 p^{5} \sigma_{\mathrm{AB}}}{256 L_{0}} \int_{0}^{d}\left[\left(\psi_{s s}-\xi^{2} \psi\right)^{2}-\omega^{2}\left(\xi^{2} \psi^{2}+\psi_{s}^{2}\right)\right] \mathrm{d} s
$$

is a function of $\omega$ and $d$ only. Since $A_{1}$ is an arbitrary constant, when $\Psi(\omega, d) \geqslant 0$, the minimum value of the free energy is 0 , which means that the undulation cannot decrease the free energy; when $\Psi(\omega, d)<0$, by making $A_{1}$ infinitely large $F_{T}$ would be $-\infty$, which means that Eq. (38) does not have a minimum and therefore cannot represent the free energy of the system in this case.

Now let us consider the case $\xi=0$, which implies that the problem reduces to two dimensions in the $y-z$ plane. The Euler-Lagrange equation Eq. (40) becomes

$$
g_{s s s s}+\omega^{2} g_{s s}=0 \text {. }
$$

The general solution is

$$
g(s)=A_{1} \cos (\omega s)+A_{2} \sin (\omega s)+A_{3}+A_{4} s,
$$

and the condition for a nontrivial solution is

$$
\sin (\omega d)=0 .
$$

Therefore, we may have undulations of the A-B interfaces when $\omega d=j \pi$, where $j$ is a positive integer. Under this condition the undulation of the $\mathrm{A}-\mathrm{B}$ interfaces is

$$
u(z)=g(s)=A_{1}[\cos (\omega s)-1],
$$

where $A_{1}$ is an arbitrary constant. We substitute Eq. (B13) into Eq. (39) and find that the free energy of an undulated A-B lamella is $F_{T}=0$, the same as the undistorted state $u(z)=0$. Therefore, in this case the undulation cannot decrease the free energy of the system. We attribute this to the omission of higher order terms in the free energy. [All these results are derived from the free energy Taylor expansion to second order, Eq. (38) or Eq. (39) when $\xi=0$.]

${ }^{1}$ P. Lambooy, T. P. Russell, G. J. Kellogg, A. M. Mayes, P. D. Gallagher, and S. K. Satija, Phys. Rev. Lett. 72, 2899 (1994).

${ }^{2}$ T. P. Russell, P. Lambooy, G. J. Kellogg, and A. M. Mayes, Physica B 213\&214, 22 (1995).

${ }^{3}$ N. Koneripalli, N. Singh, R. Levicky, F. S. Bates, P. D. Gallagher, and S. K. Satija, Macromolecules 28, 2897 (1995).

${ }^{4}$ G. J. Kellogg, D. G. Walton, A. M. Mayes, P. Lambooy, T. P. Russell, P. D. Gallagher, and S. K. Satija, Phys. Rev. Lett. 76, 2503 (1996).

${ }^{5}$ Q. Wang, Q. Yan, P. F. Nealey, and J. J. de Pablo, J. Chem. Phys. 112, 450 (2000).

${ }^{6}$ K. R. Shull, Macromolecules 25, 2122 (1992).

${ }^{7}$ M. S. Turner, Phys. Rev. Lett. 69, 1788 (1992).

${ }^{8}$ D. G. Walton, G. J. Kellogg, A. M. Mayes, P. Lambooy, and T. P. Russell, Macromolecules 27, 6225 (1994)

${ }^{9}$ G. Brown and A. Chakrabarti, J. Chem. Phys. 102, 1440 (1995).

${ }^{10}$ G. T. Pickett and A. C. Balazs, Macromolecules 30, 3097 (1997).

${ }^{11}$ M. W. Matsen, J. Chem. Phys. 106, 7781 (1997).

${ }^{12}$ N. Koneripalli, R. Levicky, F. S. Bates, J. Ankner, H. Kaiser, and S. K. Satija, Langmuir 12, 6681 (1996).

${ }^{13}$ E. Huang, T. P. Russell, C. Harrison, P. M. Chaikin, R. A. Register, C. J. Hawker, and J. Mays, Macromolecules 31, 7641 (1998).

${ }^{14}$ M. Kikuchi and K. Binder, Europhys. Lett., 21, 427 (1993).

${ }^{15}$ M. Kikuchi and K. Binder, J. Chem. Phys., 101, 3367 (1994).

${ }^{16}$ J. U. Sommer, A. Hoffmann, and A. Blumen, J. Chem. Phys. 111, 3728 (1999).

${ }^{17}$ T. Geisinger, M. Muller, and K. Binder, J. Chem. Phys. 111, 5241 (1999).

${ }^{18}$ T. Geisinger, M. Muller, and K. Binder, J. Chem. Phys. 111, 5251 (1999).

${ }^{19}$ G. G. Pereira and D. R. M. Williams, Macromolecules 32, 1661 (1999).

${ }^{20}$ L. Rockford, Y. Liu, P. Mansky, T. P. Russell, M. Yoon, and S. G. J. Mochrie, Phys. Rev. Lett. 82, 2602 (1999).

${ }^{21}$ Q. Wang, Q. Yan, P. F. Nealey, and J. J. de Pablo, Macromolecules (in press).

${ }^{22}$ A. Halperin, J. U. Sommer, and M. Daoud, Europhys. Lett. 29, 297 (1995).

${ }^{23}$ H. Chen and A. Chakrabarti, J. Chem. Phys. 108, 6897 (1998).

${ }^{24}$ A. Chakrabarti and H. Chen, J. Polym. Sci., Part B: Polym. Phys. 36, 3127 (1998).

${ }^{25}$ D. Petera and M. Muthukumar, J. Chem. Phys. 107, 9640 (1997).

${ }^{26}$ D. Petera and M. Muthukumar, J. Chem. Phys. 109, 5101 (1998).

${ }^{27}$ G. G. Pereira and D. R. M. Williams, Phys. Rev. Lett. 80, 2849 (1998).

${ }^{28}$ G. G. Pereira and D. R. M. Williams, Macromolecules 31, 5904 (1998)

${ }^{29}$ G. G. Pereira and D. R. M. Williams, Macromolecules 32, 758 (1999).

${ }^{30}$ S. K. Nath, P. F. Nealey, and J. J. de Pablo, J. Chem. Phys. 110, 7483 (1999).

${ }^{31}$ A. N. Semenov, Sov. Phys. JETP, 61, 733 (1985) [Zh. Eksp. Teor. Fiz. 88, $1242(1985)]$.

${ }^{32}$ T. Ohta and K. Kawasaki, Macromolecules 19, 2621 (1986).

${ }^{33}$ Z.-G. Wang and S. A. Safran, J. Phys. (France) 51, 185 (1990).

${ }^{34}$ Z.-G. Wang and S. A. Safran, J. Chem. Phys. 94, 679 (1991).

${ }^{35}$ Z.-G. Wang, J. Chem. Phys. 100, 2298 (1994).

${ }^{36}$ The phenomenological theory predicts that for thin films confined between two neutral surfaces, unfrustrated parallel lamellae and perpendicular lamellae (both having a bulk period $L_{0}$ ) have the same free energy, while experiments (Ref. 13) Monte Carlo simulations (Refs. 5,16) and selfconsistent field calculations (Refs. 10,11,17) all indicate that the perpendicular lamellae are preferred over the parallel lamellae between two neutral surfaces.

${ }^{37}$ G. G. Pereira and D. R. M. Williams, Langmuir 15, 2125 (1999).

${ }^{38}$ G. G. Pereira and D. R. M. Williams, Europhys. Lett. 44, 304 (1998). 
${ }^{39}$ T. Hashimoto, M. Shibayama, and H. Kawai, Macromolecules 13, 1237 (1980).

${ }^{40}$ F. A. Escobedo and J. J. de Pablo, J. Chem. Phys. 105, 4391 (1996).

${ }^{41}$ G. G. Pereira and D. R. M. Williams, Phys. Rev. E 60, 5841 (1999).

${ }^{42}$ Strictly speaking, Eq. (21) only represents the hard surface confinement $a t$ the surfaces, but not near the surfaces. According to the assumptions that chain trajectories are perpendicular to the A-B interface and that chains terminate at the interfaces between adjacent lamellae, the corresponding boundary conditions for the lower hard surface should be

$$
\left\{\begin{array}{l}
D_{\mathrm{B}} \sin \theta \leqslant z \text { if } \theta(z)>0 \\
D_{\mathrm{A}} \sin (-\theta) \leqslant z \text { if } \theta(z)<0
\end{array}\right.
$$

and for the upper hard surface,

$$
\left\{\begin{array}{l}
D_{\mathrm{A}} \sin \theta \leqslant D-z \quad \text { if } \theta(z)>0 \\
D_{\mathrm{B}} \sin (-\theta) \leqslant D-z \quad \text { if } \theta(z)<0
\end{array}\right. \text {. }
$$

We find that the solution using Eq. (21) may not satisfy the above equations in some regions in the case of relatively strong surface preference, for example, the case of $\delta_{\mathrm{H}}=-1\left(\delta_{\mathrm{S}}=2\right.$ and $\left.d=4\right)$ studied in Sec. III B 1 and in Ref. 19. In Fig. 6 such a region is shown by a thick dashed line. Note, however, that perpendicular lamellae are not actually preferred in this case. Therefore, this problem might be of little practical significance; the fixed boundary conditions Eq. (21) could be sufficient for the solution to satisfy the above equations when perpendicular lamellae are actually preferred. 